\title{
Revised and improved value of the QED tenth-order electron anomalous magnetic moment
}

\author{
Tatsumi Aoyama, ${ }^{1,2}$ Toichiro Kinoshita, ${ }^{3,4}$ and Makiko $\mathrm{Nio}^{2}$ \\ ${ }^{1}$ Yukawa Institute for Theoretical Physics, Kyoto University, Kyoto 606-8502, Japan \\ ${ }^{2}$ Nishina Center, RIKEN, Wako 351-0198, Japan \\ ${ }^{3}$ Laboratory for Elementary Particle Physics, Cornell University, Ithaca, New York 14853, USA \\ ${ }^{4}$ Amherst Center for Fundamental Interactions, Department of Physics, University of Massachusetts, \\ Amherst, Massachusetts 01003, USA
}

(Received 18 December 2017; published 8 February 2018)

\begin{abstract}
In order to improve the theoretical prediction of the electron anomalous magnetic moment $a_{e}$ we have carried out a new numerical evaluation of the 389 integrals of Set $\mathrm{V}$, which represent 6,354 Feynman vertex diagrams without lepton loops. During this work, we found that one of the integrals, called X024, was given a wrong value in the previous calculation due to an incorrect assignment of integration variables. The correction of this error causes a shift of -1.26 to the Set V contribution, and hence to the tenth-order universal (i.e., mass-independent) term $A_{1}^{(10)}$. The previous evaluation of all other 388 integrals is free from errors and consistent with the new evaluation. Combining the new and the old (excluding X024) calculations statistically, we obtain $7.606(192)(\alpha / \pi)^{5}$ as the best estimate of the Set V contribution. Including the contribution of the diagrams with fermion loops, the improved tenth-order universal term becomes $A_{1}^{(10)}=6.675(192)$. Adding hadronic and electroweak contributions leads to the theoretical prediction $a_{e}$ (theory) $=1159652182.032(720) \times 10^{-12}$. From this and the best measurement of $a_{e}$, we obtain the inverse fine-structure constant $\alpha^{-1}\left(a_{e}\right)=137.0359991491(331)$. The theoretical prediction of the muon anomalous magnetic moment is also affected by the update of QED contribution and the new value of $\alpha$, but the shift is much smaller than the theoretical uncertainty.
\end{abstract}

DOI: 10.1103/PhysRevD.97.036001

\section{INTRODUCTION AND SUMMARY}

In 1947 the electron magnetic moment anomaly $a_{e}=$ $(g-2) / 2$ was discovered in an atomic physics experiment [1], which was soon understood as the effect of radiative correction by the newly formulated quantum electrodynamics (QED) [2]. Since then comparison of measurement and theory of $a_{e}$ has provided more and more stringent test of QED and the standard model (SM) of elementary particles.

The most accurate measurement of $a_{e}$ thus far has been carried out by the Harvard group using a cylindrical Penning trap [3,4]:

$$
a_{e}(\mathrm{HV} 08)=1159652180.73(28) \times 10^{-12}[0.24 \mathrm{ppb}] .
$$

Published by the American Physical Society under the terms of the Creative Commons Attribution 4.0 International license. Further distribution of this work must maintain attribution to the author(s) and the published article's title, journal citation, and DOI. Funded by SCOAP .
The precision of this value is fifteen times higher than that of the pioneering work by the group at the University of Washington [5]. Further improvements for the electron and positron measurements are currently being prepared by the Harvard group [6].

To test the validity of the theory of $a_{e}$, it must be evaluated to match the precision of the measurement (1). The dominant contribution comes from QED, while at such a precision, the SM contribution can no longer be ignored. Thus we can write

$$
a_{e}=a_{e}(\mathrm{QED})+a_{e}(\text { Hadron })+a_{e}(\text { Weak }) .
$$

The QED contribution can be expressed further, by taking the heavier leptons ( $\mu$ and $\tau$ ) into account, as

$$
\begin{aligned}
a_{e}(\mathrm{QED})= & A_{1}+A_{2}\left(m_{e} / m_{\mu}\right)+A_{2}\left(m_{e} / m_{\tau}\right) \\
& +A_{3}\left(m_{e} / m_{\mu}, m_{e} / m_{\tau}\right) .
\end{aligned}
$$

Note that the mass-dependence appears in the form of mass ratio because $a_{e}$ is dimensionless. All four terms are expressed in the perturbation series of the fine-structure constant $\alpha$ 


$$
A_{n}=\sum_{i=1,2, \cdots}\left(\frac{\alpha}{\pi}\right)^{i} A_{n}^{(2 i)}
$$

Since the electron is the lightest lepton, contributions from heavier particles are suppressed and tiny, although not negligible.

The QED contribution involving heavy leptons are known with sufficient precision. The muon and tau-lepton contributions $A_{2}$ and $A_{3}$ of $a_{e}$ up to eighth order have been calculated both numerically and analytically, with a good agreement with each other [7-15]. The tenth-order massdependent contribution $A_{2}^{(10)}\left(m_{e} / m_{\mu}\right)$ has been evaluated numerically [16-25]. Some of the tenth-order diagrams have been independently calculated and checked [26]. The tau-lepton contribution to the tenth-order term is currently negligible compared to the $A_{1}$ term since it is suppressed by the factor $\left(m_{e} / m_{\tau}\right)^{2}$ and contributes to $a_{e}$ no more than $\mathcal{O}\left(10^{-18}\right)$. Summing all mass-dependent terms, we obtain

$a_{e}(\mathrm{QED}:$ mass-dependent $)=2.7475719(13) \times 10^{-12}$,

where the uncertainty comes from the tau-electron mass ratio. The uncertainty due to the muon-electron mass ratio is one order of magnitude smaller and is about $0.13 \times 10^{-18}$.

To compare the theory with the experiment, it is of course necessary to evaluate the mass-independent term $A_{1}$ up to tenth order of perturbation theory, since $(\alpha / \pi)^{5} \sim 0.07 \times 10^{-12}$. The second-, fourth-, and sixthorder terms were calculated analytically [2,27-29] or by numerical or semianalytical means $[30,31]$ :

$$
\begin{gathered}
A_{1}^{(2)}=0.5, \\
A_{1}^{(4)}=-0.328478965579193 \ldots \\
A_{1}^{(6)}=1.181241456587 \ldots
\end{gathered}
$$

Recently, Laporta has reported a highly precise value of the eighth-order term $A_{1}^{(8)}$, with an accuracy of 1100 digits, after twenty years of persistent research [32]. Thus the uncertainty due to the eighth-order term has been completely eliminated by his outstanding work. It took 36 years since the preliminary value $A_{1}^{(8)}=-0.8(2.5)$ was reported [33]. For the purpose of this article it is sufficient to list the first ten digits of Laporta's result:

$$
A_{1}^{(8)}[\text { semi-analytic }]=-1.912245764 \ldots,
$$

which confirms the validity of the earlier numerical evaluation [34]

$$
A_{1}^{(8)}[\text { numerical }]=-1.91298(84) .
$$

Although it is less accurate than (10), another semianalytic result $A_{1}^{(8)}=-1.87$ (12) given in Ref. [35] is consistent with both of preceding results (9) and (10). The contribution to $A_{1}^{(8)}$ from the 518 vertex diagrams without a fermion loop has also been independently cross-checked by using numerical means [36].

The tenth-order mass-independent term $A_{1}^{(10)}$ is thus the only significant QED contribution which has not been verified by independent calculations. It has a contribution from 12,672 vertex-type Feynman diagrams. Of these diagrams those that are dominant and the hardest to evaluate belong to Set $\mathrm{V}$, which consists of 6,354 vertex diagrams without a fermion loop. Two of these vertex diagrams have been evaluated by other means thus far. Their values are given in Refs. [36,37]. We have compressed all 6,354 vertex diagrams to 389 integrals by certain algebraic manipulation. The preliminary value of the contribution of Set $\mathrm{V}$ obtained by numerical integration by VEGAS was $[34,38]$

$$
A_{1}^{(10)}[\operatorname{Set} \mathrm{V}: 2015]=8.723(336) .
$$

In order to improve this further, we have reevaluated these 389 integrals using independent sets of integration variables. The result is

$$
A_{1}^{(10)}[\text { Set V: 2017] }=7.791(264),
$$

which disagrees with (11) by -0.93 .

This discrepancy arises mainly from the integral X024 expressing the contribution from Fig. 1, which represents the sum of nine vertex diagrams. During the new evaluation we found a programming error in the previous evaluation of $X 024$. When the error was corrected, its numerical value shifted from -6.0902 (246) to -7.3480 (139). The difference of -1.26 accounts for almost all the difference between the values (11) and (12). If this correction of $X 024$ is added to the old result (11), we obtain

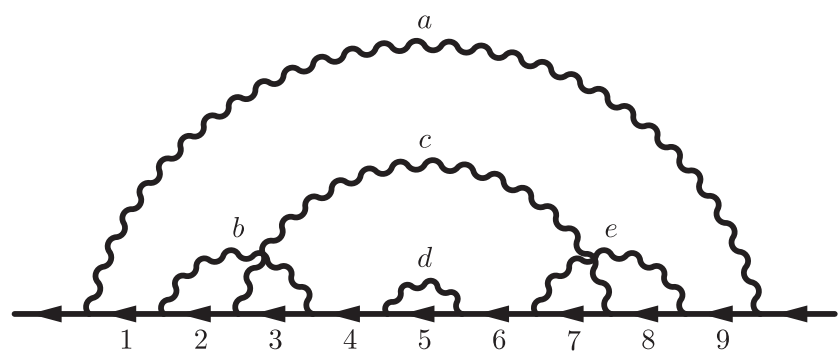

FIG. 1. Self-energy-like diagram X024(abcbddecea). The straight and wavy lines represent fermion and photon propagators, respectively. Indices assigned to the fermion lines are $1,2, \ldots, 9$ from left to right, and those to the photon lines are $a, b, \ldots, e$. The nine vertex diagrams related to this self-energylike diagram are obtained by inserting an external photon vertex in each of the nine fermion lines. 


$$
A_{1}^{(10)}[\text { Set V : } 2015 \text { corrected }]=7.465(335),
$$

which is consistent with the new value of Set $\mathrm{V}$ given in Eq. (12). The details of the origin of the error in the X024 integral are discussed in Sec. V.

Since the new calculation (12) is independent of the old one, two calculations can be statistically combined for each integral. The best estimate for Set V then becomes

$$
A_{1}^{(10)}[\text { Set V }]=7.606(192) \text {. }
$$

Adding the contribution from the 6,318 diagrams with fermion loops [16-25]

$$
A_{1}^{(10)}[\text { Set I-IV, VI }]=-0.93042(361)
$$

to (14), we obtain an updated value of the tenth-order massindependent contribution:

$$
A_{1}^{(10)}=6.675(192),
$$

where the uncertainty comes entirely from the numerical integration of Set $\mathrm{V}$ and is reduced by $43 \%$ compared to that in (11). This is the main result of our paper.

The contributions of the electroweak interaction and the hadronic interaction have been updated recently [39] including new hadronic measurements $[40,41]$ :

$$
\begin{aligned}
a_{e}(\text { Weak })= & 0.03053(23) \times 10^{-12}, \\
a_{e}(\text { Hadron })= & \{1.8490(108)-0.2213(12)+0.0280(2) \\
& +0.037(5)\} \times 10^{-12} \\
= & 1.6927(120) \times 10^{-12},
\end{aligned}
$$

respectively, where the hadronic contribution consists of the leading-order (LO), next-to-leading-order (NLO), and next-to-next-to-leading-order (NNLO) vacuum-polarization (VP) contributions and the hadronic light-by-light scattering contribution from left to right. The combined uncertainty of $a_{e}$ (Hadron) is the one given in Eq. (5) of Ref. [39].

It is noted that the same spectral function is used to obtain the LO-, NLO-, and NNLO-VP contributions, and their systematic uncertainties are correlated, as pointed out in Refs. [42,43]. The correlation should be taken into account to derive the combined uncertainty (17).

Summing up all the contributions of SM, we obtain the theoretical prediction for $a_{e}$ as

$a_{e}$ (theory) $=1159652182.032(13)(12)(720) \times 10^{-12}$,

where the first and second uncertainties are due to the tenth-order QED and the hadronic corrections, respectively.
The uncertainty due to the mass ratios of tau or muon to electron is currently negligible. The third and largest uncertainty comes from the fine-structure constant $\alpha$. Here, we used the latest value of $\alpha[44,45]$, determined from the recoil measurement of the Rb atom $h / M_{\mathrm{Rb}}$ [46] combined with the relative atomic mass of the electron $A(e)$ from the $g$-factor of the bound electron [44], the relative atomic mass of the $\mathrm{Rb}$ atom $A(\mathrm{Rb})$ [47,48], and the Rydberg constant $R_{\infty}$ [44]:

$$
\alpha^{-1}(\mathrm{Rb}: 2016)=137.035998995(85)
$$

which replaces the value given in Ref. [49], $\alpha^{-1}(\mathrm{Rb}: 2010)=137.035999049(90)$.

The new theoretical value of $a_{e}$ is greater by $0.38 \times$ $10^{-12}$ than that of Eq. (16) of Ref. [34]. The corrected and updated $A_{1}^{(10)}$ adds $-0.08 \times 10^{-12}$ to $a_{e}$, the near-exact value of $A_{1}^{(8)}$ increases $a_{e}$ by $0.02 \times 10^{-12}$, and the new value $\alpha(\mathrm{Rb})$ increases $a_{e}$ by $0.45 \times 10^{-12}$. The shift due to the new values of the electroweak and hadronic contributions is $-0.01 \times 10^{-12}$. The difference between experiment (1) and theory (18) is thus

$a_{e}(\mathrm{HV} 08)-a_{e}($ theory $)=(-1.30 \pm 0.77) \times 10^{-12}$.

If we assume that the theory of $a_{e}$ is correct, by equating the formula Eq. (18) to the measured value Eq. (1), we obtain an $\alpha$ which is more precise than that of (19):

$\alpha^{-1}\left(a_{e}: 2017\right)=137.0359991491(15)(14)(330)$,

where the uncertainties come from the tenth-order QED, the hadronic correction, and the experiment. The shift from the previous value given in Eq. (18) of Ref. [34] amounts to $-0.87 \times 10^{-8}$ and is due to the new values of $A_{1}^{(8)}, A_{1}^{(10)}$, and the electroweak and hadronic corrections. The difference between two determinations (21) and (19) of $\alpha$ is

$$
\alpha^{-1}\left(a_{e}: 2017\right)-\alpha^{-1}(\mathrm{Rb}: 2016)=(0.155 \pm 0.091) \times 10^{-6} .
$$

Since the updated QED contributions of $A_{1}^{(8)}$ and $A_{1}^{(10)}$ are universal for any lepton species, the theoretical prediction of the muon anomalous magnetic moment $\left(a_{\mu}\right)$ should also be changed. The new value of the fine-structure constant $\alpha$ derived from $a_{e}$ also causes a small shift in $a_{\mu}$. The total shift caused by them is, however, far smaller than the current theoretical uncertainty and has no significant influence on comparison of theory and experiment of $a_{\mu}$. The details of the updates of $a_{\mu}$ are given in the Appendix. 


\section{PREPARATION OF SET V FOR NUMERICAL EVALUATION}

QED is renormalizable. But individual Feynman diagrams are divergent. For numerical integration, it is absolutely necessary that integrals do not contain any divergence. This means that all divergences must be removed before integration is carried out.

Another problem with Set V, which consists of 6,354 tenth-order vertex diagrams without a fermion loop, is its huge size. It should be difficult to achieve high precision in the numerical evaluation because of accumulation of uncertainties of individual diagrams, even though each diagram were evaluated precisely enough. Thus it is highly desirable to reduce the number of independent integrals as much as possible. One way to achieve such reduction is to sum up 9 vertex diagrams obtained by inserting an external photon vertex in each of 9 fermion lines in a tenth-order electron self-energy diagram which does not contain a closed fermion loop. Let $\Sigma(p)$ be such a self-energy diagram and $\Lambda^{\nu}(p, q)$ the sum of 9 vertex diagrams related to $\Sigma(p)$. Then, with the help of an equation derived from the Ward-Takahashi identity, one finds

$$
\Lambda^{\nu}(p, q) \approx q_{\mu}\left[\frac{\partial \Lambda^{\mu}(p, q)}{\partial q_{\nu}}\right]_{q=0}-\frac{\partial \Sigma(p)}{\partial p_{\nu}}
$$

in the small $q$ limit. The sum of all vertex diagrams of Set $\mathrm{V}$ can then be represented by 706 quantities of the form given on the right hand side of Eq. (23), which we shall call "selfenergy-like" diagram. Taking the time-reversal symmetry into account, we can reduce it further to 389 self-energylike diagrams which represent all vertex diagrams of Set V.

We assign Feynman parameters $z_{1}, \ldots, z_{9}$ to nine fermion lines and $z_{a}, \ldots, z_{e}$ to five photon lines from the left to the right. They are subject to the constraint $z_{1}+\cdots+z_{9}+$ $z_{a}+\cdots+z_{e}=1$.

Each self-energy-like diagram is then represented by a sequence of ten vertices on the fermion line labeled by the photon Feynman parameters. For instance, X024 in Fig. 1 is represented by the sequence "abcbddecea."

The integral for a self-energy-like diagram $\mathcal{G}$ is defined in the Feynman parameter space and has a form

$$
\begin{aligned}
M_{\mathcal{G}}= & \left(\frac{-1}{4}\right)^{5} 4 ! \int(d z)_{\mathcal{G}} \\
& \times\left[\frac{1}{4}\left(\frac{E_{0}+C_{0}}{U^{2} V^{4}}+\frac{E_{1}+C_{1}}{U^{3} V^{3}}+\cdots+\frac{E_{3}+C_{3}}{U^{5} V}\right)\right. \\
& \left.+\left(\frac{N_{0}+Z_{0}}{U^{2} V^{5}}+\frac{N_{1}+Z_{1}}{U^{3} V^{4}}+\cdots+\frac{N_{4}+Z_{4}}{U^{6} V}\right)\right],
\end{aligned}
$$

where $(d z)_{\mathcal{G}}=d z_{1} \ldots d z_{9} d z_{a} \ldots d z_{e} \delta\left(1-z_{1} \ldots-z_{9}-z_{a} \ldots\right.$ $\left.-z_{e}\right)$, all $z_{i}$ being non-negative. $E_{n}$ and $C_{n}$ terms and $N_{n}$ and $Z_{n}$ terms are projected out from the first and second terms of the right-hand side of Eq. (23), respectively. All functions $E_{n}, C_{n}, N_{n}, Z_{n}, V, U$ are expressed in terms of the Feynman parameters and the building blocks $A_{i}, B_{i j}, C_{i j}$ for $i, j=1,2, \ldots, 9$, which are polynomials of Feynman parameters. The detailed definitions of these functions are given in Refs. [50-52].

The bare amplitude $M_{\mathcal{G}}$ is inherently ultraviolet (UV) divergent. If the diagram $\mathcal{G}$ possesses a self-energy subdiagram, it suffers from infrared (IR) divergence as well. Though we do not explicitly write them, the amplitude $M_{\mathcal{G}}$ is regularized by the Feynman cutoff for UV divergence and the small photon mass for IR divergence. Both regulators are safely and harmlessly removed after the finite integral is constructed with the bare amplitude $M_{\mathcal{G}}$ and the corresponding UV and IR counterterms.

The UV divergence emerges with a self-energy or vertex subdiagram, and the entire divergence structure is obtained in the form of Zimmermann's forests [53]. The $\boldsymbol{K}$-operation, which extracts the most UV-divergent part of a subdiagram, is applied to $M_{\mathcal{G}}$ according to Zimmermann's forest formula, and the UV subtraction terms are generated $[52,54]$. For IR divergences, the divergent structure is given by the annotated forests of self-energy subdiagrams to which are assigned either magnetic moment or self-mass properties. Either the $\boldsymbol{I}$ - or $\boldsymbol{R}$-subtraction operation is applied to $M_{\mathcal{G}}$ according to the annotation to generate the IR subtraction terms [34,55]. UV divergence arising in the IR subtraction terms are further subtracted by applying the $\boldsymbol{K}$-operation to them.

The finite magnetic moment amplitude $\Delta M_{\mathcal{G}}$ as the output of these operations on the diagram $M_{\mathcal{G}}$, made UV-finite by $\boldsymbol{K}$-operation and IR-finite by $\boldsymbol{R}$ - and/or $I$-operations, is thus symbolically written as

$$
\begin{aligned}
\Delta M_{\mathcal{G}}= & M_{\mathcal{G}}+\sum_{\text {forests }}\left(-\boldsymbol{K}_{S_{i}}\right) \cdots\left(-\boldsymbol{K}_{S_{j}}\right) M_{\mathcal{G}} \\
& +\sum_{\text {annotated forests }}\left(-\boldsymbol{I}_{S_{i}}\right) \cdots\left(-\boldsymbol{I}_{S_{j}}\right)\left(-\boldsymbol{R}_{S_{k}}\right) \cdots\left(-\boldsymbol{R}_{S_{l}}\right) M_{\mathcal{G}}
\end{aligned}
$$

The sum over all diagrams is denoted as $\Delta M_{10}$

$$
\Delta M_{10}=\sum_{\mathcal{G}=X 001}^{X 389} \Delta M_{\mathcal{G}},
$$

where the time-reversal symmetric factor 2 is included in the definition of $\Delta M_{\mathcal{G}}$.

The procedure to generate the finite amplitude can be handled in an automated way by the code-generating program GENCODE $N$. It takes the one-line expression of a diagram $\mathcal{G}$ using a sequence of photon labels on the fermion line and creates the form of the bare amplitude. The generator then identifies the divergent subdiagrams and their forests, and constructs the corresponding UV and IR 
subtraction terms. Finally, the finite amplitude ready to be integrated by the numerical integration routine is generated [34,55]. Numerical integration of individual $\Delta M_{\mathcal{G}}$ is described in the next section.

\section{NUMERICAL INTEGRATION OF $\Delta M_{10}$ BY VEGAS}

Because of the linear constraint imposed on the 14 Feynman parameters, the integration domain $(d z)_{\mathcal{G}}$ is a 13 dimensional hyperplane. For a diagram which has no self-energy subdiagrams, the 14 Feynman parameters of the integrand are mapped onto a 13 dimensional unit hypercube that is the integration domain of the integration algorithm VEGAS. There are many ways to realize the mapping from the 14 Feynman parameters to the 13 integration variables. Analytically any choices of mapping, of course, give the same value.

For a diagram with a self-energy subdiagram, we can reduce the number of integration variables. After projecting out the magnetic moment contribution from a self-energy-like diagram, the integrand depends only on the sum of the adjacent Feynman parameters $z_{i-1}+z_{j+1}$, if the diagram contains a self-energy subdiagram $S_{i j}$ containing the fermion lines $\{i, i+1, \ldots, j-1, j\}$ from the left to the right. In the case of X024 (abcbddecea), it contains two self-energy subdiagrams $S_{28}$ and $S_{55}$ so that the integrand of $X 024$ depends on the sums $z_{19} \equiv z_{1}+z_{9}$ and $z_{46} \equiv z_{4}+z_{6}$. The $X 024$ can then be numerically evaluated on a 11-dimensional hypercube instead of a 13-dimensional one.

Such reduction of independent variables is related to the loop topology of the Feynman diagram. The adjacent fermion lines attached to a self-energy subdiagram can be regarded topologically as the same line. Since they are determined by and only by the loop topology of a diagram, the building blocks $B_{i-1, k}$ and $B_{j+1, k}$ for any $k$ have exactly the same expression if the indices $i-1$ and $j+1$ belong to the adjacent fermion lines of the same self-energy subdiagram $S_{i j}$. The scalar currents $A_{i}$ of the adjacent fermion lines are also exactly the same because the vanishing limit of the transfer momentum $(q \rightarrow 0)$ is taken when the magnetic moment contribution is projected out. For the case of $X 024$, the adjacent fermion lines $z_{1}$ and $z_{9}$ lead to $B_{1 k}=B_{k 9}\left(=B_{k 1}=B_{9 k}\right)$ for any $k$ of the fermion and photon lines. For the scalar currents, $A_{1}=A_{9}$. Implementation to these features into GENCODE $N$ is straightforward. The resulting integrand becomes much shorter, almost halved in many cases, that enables us to reduce the computational cost significantly.

Among the 389 self-energy-like diagrams of Set V, the 254 diagrams have at least one self-energy subdiagram. They are grouped as $X B$, and have been evaluated with dimensions from 9 to 12 , depending on the numbers of selfenergy subdiagrams involved. The remaining 135 diagrams that are grouped as $X L$ receive only vertex corrections, and have been evaluated with 13 dimensions.

An $X B$ integrand has at least one IR subtraction term. The cancellation of the IR divergence within the integrand is in the form of the inverse power law of the vanishing photon mass. Some of the $X B$ integrals suffer from very severe round-off errors for finite numerical precision, and the VEGAS integration breaks down while the integration is iterated. The simplest solution to this digit-deficiency problem is to use an extended precision for real numbers. In order to accelerate the convergence of numerical calculation on a computer, an algorithm that realizes doubledouble (DD) precision [56] has been adopted. The arrayed version of the DD library was prepared by one of us (T. A.) and used for productive execution. The 253 of $254 X B$ integrals have been evaluated with the DD precision in the entire integration domain.

The diagram X008 (abbccddeea) suffers from the most severe IR cancellation, and needs the quadruple-double (QD) precision for real numbers [56] in some part of the integration domain. We divided the entire integration domain of X008 into four, and applied the QD precision for the most dangerous but narrow region. The DD precision is used for the regions including the dangerous edges. In the remaining wide region, the shape of the integrand $X 008$ is relatively smooth, and the double precision is sufficient to carry out the integration. The same division rule were also adopted for the previous calculation. We moved the borders between four regions slightly in the new calculation.

Unlike IR divergences, UV divergence is in powers of logarithms at most. The double precision for real numbers implemented in a standard hardware is sufficient to handle the UV cancellation. The $X L$ integrals involve no IR subtraction terms and have only UV subtraction terms. They were therefore evaluated in double precision.

It is found that the elapsed wall-clock time needed for VEGAS to perform one iteration of an integration strongly depends on the array parameter of the arrayed DD library. After many trial runs, we determined the array parameters for computers of three different architectures to make numerical computation of Set $\mathrm{V}$ as fast as possible.

We follow the next procedure for a VEGAS integration: We start from a flat grid structure. The grid is adjusted automatically after each iteration with $10^{7}$ sampling points, that is iterated for 50 times. Then the number of sampling points is increased to $10^{8}$ in several steps, and additional 50 iterations are carried out. After a good grid structure is formed, the productive calculation starts with sampling points $2.56 \times 10^{9}$ or $4 \times 10^{9}$ per iteration, depending on the difficulty of the integration. The iteration is repeated at least 2 times, until the estimated uncertainty of VEGAS for the integration result decreases to less than 0.025 .

The twelve integrals showing slow convergence and larger uncertainties were evaluated once more with different mappings on a new computer system, 
TABLE I. VEGAS integration results of X001-X389 of the tenth-order Set V diagrams. The first and second columns show the diagram name and its representation in terms of photon indices, respectively. The third and fourth columns list the VEGAS integration results used in Ref. [34] and the new VEGAS integration result of this work, respectively. An uncertainty of the integral corresponds to a $1.65 \sigma$, which is $90 \%$ confidence level, determined by VEGAS assuming gaussian distribution of statistical ensembles for the integral. The difference of two results is listed in the fifth column. The weighted average of the third and fourth columns is listed in the last column. The old result of $X 024$ from Ref. [34] is incorrect and is removed from the third column. The integral X024 is thus not averaged over the third and fourth columns.

\begin{tabular}{|c|c|c|c|c|c|}
\hline Diagram $\mathcal{G}$ & Expression & Value (Error) in Ref. [34] & Value (Error) in this work & Difference & Weighted average \\
\hline$X 001$ & abacbdcede & $-0.1724(92)$ & $-0.1591(35)$ & -0.0133 & $-0.1608(33)$ \\
\hline$X 002$ & abaccddebe & $-5.9958(333)$ & $-5.9488(176)$ & -0.0471 & $-5.9591(156)$ \\
\hline$X 003$ & abacdbcede & $-0.1057(52)$ & $-0.1048(18)$ & -0.0009 & $-0.1049(17)$ \\
\hline$X 004$ & abacdcdebe & $5.1027(339)$ & $5.1019(182)$ & 0.0007 & $5.1021(160)$ \\
\hline$X 005$ & abacddbece & $1.1112(168)$ & $1.0973(212)$ & 0.0138 & $1.1058(131)$ \\
\hline$X 006$ & abacddcebe & $-5.2908(245)$ & $-5.2942(215)$ & 0.0033 & $-5.2927(161)$ \\
\hline$X 007$ & abbcadceed & $-3.4592(254)$ & $-3.4319(217)$ & -0.0273 & $-3.4434(165)$ \\
\hline$X 008$ & $a b b c c d d e e a$ & $-16.5070(289)$ & $-16.4999(201)$ & -0.0071 & $-16.5022(165)$ \\
\hline$X 009$ & abbcdaceed & $-3.1069(71)$ & $-3.1336(174)$ & 0.0267 & $-3.1107(65)$ \\
\hline$X 010$ & $a b b c d c d e e a$ & $11.2644(342)$ & $11.2817(178)$ & -0.0173 & $11.2780(158)$ \\
\hline$X 011$ & $a b b c d d a e e c$ & $6.0467(338)$ & $6.0445(183)$ & 0.0022 & $6.0450(161)$ \\
\hline$X 012$ & $a b b c d d c e e a$ & $-9.3328(267)$ & $-9.3587(211)$ & 0.0259 & $-9.3488(166)$ \\
\hline$X 013$ & abcabdecde & -1.3710 & -1.3759 (9) & 0.0049 & $-1.3755(9)$ \\
\hline$X 014$ & abcacdedbe & $0.8727(42)$ & $0.8789(15)$ & -0.0062 & $0.8782(14)$ \\
\hline$X 015$ & abcadbecde & $2.1090(8)$ & 2.1107 (4) & -0.0017 & $2.1103(4)$ \\
\hline$X 016$ & abcadcedbe & $-0.9591(7)$ & -0.9588 & -0.0003 & -0.9588 \\
\hline$X 017$ & abcaddebce & $0.5146(13)$ & $0.5162(20)$ & -0.0016 & $0.5151(11)$ \\
\hline$X 018$ & abcaddecbe & 0.0309 (13) & $0.0323(21)$ & -0.0014 & 0.0313 \\
\hline$X 019$ & abcbadeced & $1.2965(48)$ & $1.3028(15)$ & -0.0063 & $1.3022(14)$ \\
\hline$X 020$ & $a b c b c d e d e a$ & $-8.1900(318)$ & $-8.1534(187)$ & -0.0366 & $-8.1628(161)$ \\
\hline$X 021$ & abcbdaeced & $-0.2948(15)$ & -0.2954 (9) & 0.0006 & $-0.2952(8)$ \\
\hline$X 022$ & abcbdcedea & $0.8892(226)$ & $0.8839(211)$ & 0.0053 & 0.8864 (154) \\
\hline$X 023$ & $a b c b d d e a e c$ & $0.4485(55)$ & $0.4543(103)$ & -0.0058 & $0.4498(49)$ \\
\hline$X 024$ & abcbddecea & - & $-7.3481(139)$ & - & $-7.3481(139)$ \\
\hline$X 025$ & abccadeebd & -0.7482 (194) & -0.7585 & 0.0103 & $-0.7528(145)$ \\
\hline$X 026$ & $a b c c b d e e d a$ & $-7.8258(277)$ & $-7.8213(210)$ & -0.0045 & $-7.8230(167)$ \\
\hline$X 027$ & abccdaeebd & $-2.3260(54)$ & $-2.3185(68)$ & -0.0075 & $-2.3231(42)$ \\
\hline$X 028$ & abccdbeeda & $4.5663(342)$ & $4.5459(177)$ & 0.0204 & $4.5502(157)$ \\
\hline X029 & $a b c c d d e e a b$ & $6.9002(233)$ & $6.9066(183)$ & -0.0064 & $6.9042(144)$ \\
\hline$X 030$ & $a b c c d d e e b a$ & $-12.6225(342)$ & -12.6385 (193) & 0.0160 & $-12.6346(168)$ \\
\hline$X 031$ & $a b c d a e b c d e$ & $2.3000(14)$ & $2.3011(8)$ & -0.0011 & $2.3009(6)$ \\
\hline$X 032$ & abcdaecdbe & $-0.2414(6)$ & -0.2422 & 0.0008 & -0.2420 \\
\hline$X 033$ & abcdaedbce & $-1.3806(7)$ & -1.3809 (4) & 0.0003 & -1.3808 \\
\hline$X 034$ & abcdaedcbe & 1.2585 (9) & $1.2599(5)$ & -0.0014 & 1.2595 \\
\hline$X 035$ & abcdbeaced & -0.5899 & $-0.5899(2)$ & -0.0000 & -0.5899 \\
\hline$X 036$ & $a b c d b e c d e a$ & $0.2318(11)$ & $0.2327(22)$ & -0.0009 & $0.2320(10)$ \\
\hline$X 037$ & abcdbedaec & $-0.7407(5)$ & -0.7410 & 0.0003 & $-0.7409(2)$ \\
\hline X038 & $a b c d b e d c e a$ & $-0.2927(14)$ & $-0.2919(21)$ & -0.0008 & $-0.2924(11)$ \\
\hline$X 039$ & $a b c d c e a e b d$ & $0.3292(12)$ & $0.3307(9)$ & -0.0015 & $0.3301(7)$ \\
\hline$X 040$ & $a b c d c e b e d a$ & $1.3397(50)$ & $1.3434(66)$ & -0.0037 & $1.3411(40)$ \\
\hline$X 041$ & $a b c d c e d e a b$ & 3.1076 (94) & $3.1219(160)$ & -0.0143 & $3.1113(81)$ \\
\hline$X 042$ & $a b c d c e d e b a$ & $-4.1353(192)$ & $-4.1235(218)$ & -0.0119 & $-4.1301(144)$ \\
\hline$X 043$ & $a b c d d e e a b c$ & $-2.9620(29)$ & $-2.9633(53)$ & 0.0013 & $-2.9623(25)$ \\
\hline$X 044$ & $a b c d d e e b c a$ & $4.4121(281)$ & $4.3654(160)$ & 0.0467 & 4.3769 (139) \\
\hline$X 045$ & $a b c d d e e c a b$ & $3.4331(212)$ & $3.4338(206)$ & -0.0007 & $3.4335(148)$ \\
\hline$X 046$ & $a b c d d e e c b a$ & $-7.7564(339)$ & $-7.7945(187)$ & 0.0382 & $-7.7856(163)$ \\
\hline$X 047$ & abcdeabcde & $-4.4496(40)$ & $-4.4602(11)$ & 0.0106 & $-4.4594(11)$ \\
\hline$X 048$ & abcdeacdbe & $-0.8061(8)$ & -0.8058 & -0.0003 & -0.8058 \\
\hline$X 049$ & abcdeadbce & -0.0278 & -0.0280 & 0.0003 & -0.0280 \\
\hline
\end{tabular}


TABLE I. (Continued)

\begin{tabular}{|c|c|c|c|c|c|}
\hline Diagram $\mathcal{G}$ & Expression & Value (Error) in Ref. [34] & Value (Error) in this work & Difference & Weighted average \\
\hline$X 050$ & abcdeadcbe & $-1.2213(9)$ & $-1.2213(5)$ & -0.0000 & $-1.2213(4)$ \\
\hline$X 051$ & abcdebaced & $-0.1776(6)$ & $-0.1774(4)$ & -0.0001 & $-0.1775(3)$ \\
\hline$X 052$ & abcdebcdea & $1.0293(17)$ & $1.0279(30)$ & 0.0014 & $1.0289(15)$ \\
\hline$X 053$ & abcdebdaec & $0.3699(4)$ & $0.3702(2)$ & -0.0002 & $0.3701(2)$ \\
\hline$X 054$ & abcdebdcea & $-0.5174(11)$ & $-0.5196(20)$ & 0.0023 & $-0.5179(9)$ \\
\hline$X 055$ & abcdecaebd & $-0.3673(4)$ & $-0.3679(2)$ & 0.0006 & $-0.3677(1)$ \\
\hline$X 056$ & abcdecbeda & $-0.2650(27)$ & $-0.2608(42)$ & -0.0042 & $-0.2637(23)$ \\
\hline$X 057$ & $a b c d e c d e a b$ & $2.7370(31)$ & $2.7385(58)$ & -0.0015 & $2.7373(27)$ \\
\hline$X 058$ & $a b c d e c d e b a$ & $-5.2510(70)$ & $-5.2249(140)$ & -0.0261 & $-5.2457(63)$ \\
\hline$X 059$ & $a b c d e d e a b c$ & $2.1866(28)$ & $2.1758(50)$ & 0.0108 & $2.1841(24)$ \\
\hline$X 060$ & $a b c d e d e b c a$ & $-3.2089(188)$ & $-3.1792(216)$ & -0.0297 & $-3.1962(142)$ \\
\hline$X 061$ & $a b c d e d e c a b$ & $-3.7724(137)$ & $-3.7874(216)$ & 0.0149 & $-3.7767(116)$ \\
\hline$X 062$ & $a b c d e d e c b a$ & $5.9174(262)$ & 5.8861 (219) & 0.0313 & $5.8990(168)$ \\
\hline$X 063$ & abcdeeabcd & $3.4295(14)$ & $3.4297(26)$ & -0.0002 & $3.4296(12)$ \\
\hline$X 064$ & abcdeeacbd & $-0.2772(8)$ & -0.2779 (14) & 0.0008 & $-0.2774(7)$ \\
\hline$X 065$ & abcdeebadc & 0.1551 (13) & $0.1580(21)$ & -0.0029 & $0.1559(11)$ \\
\hline$X 066$ & $a b c d e e b c d a$ & $-3.6145(45)$ & $-3.6177(81)$ & 0.0033 & $-3.6152(39)$ \\
\hline$X 067$ & $a b c d e e c d a b$ & $-1.6761(85)$ & $-1.6853(168)$ & 0.0092 & $-1.6780(76)$ \\
\hline$X 068$ & $a b c d e e c d b a$ & 2.7855 (217) & $2.7540(205)$ & 0.0315 & 2.7689 (149) \\
\hline$X 069$ & $a b c d e e d a b c$ & $-1.2627(31)$ & $-1.2690(45)$ & 0.0063 & $-1.2647(25)$ \\
\hline$X 070$ & $a b c d e e d b c a$ & 3.2149 (144) & $3.2001(212)$ & 0.0148 & 3.2102 (119) \\
\hline$X 071$ & $a b c d e e d c a b$ & $3.7025(96)$ & $3.6943(187)$ & 0.0083 & $3.7008(85)$ \\
\hline$X 072$ & $a b c d e e d c b a$ & $-5.5704(208)$ & $-5.5658(209)$ & -0.0047 & $-5.5681(147)$ \\
\hline$X 073$ & abacbdceed & 3.4114 (254) & 3.3929 (212) & 0.0184 & 3.4005 (162) \\
\hline$X 074$ & abacbddece & $4.4104(251)$ & 4.3889 (212) & 0.0215 & $4.3978(162)$ \\
\hline$X 075$ & abacbddeec & $-8.1138(340)$ & $-8.0608(195)$ & -0.0531 & -8.0739 (169) \\
\hline$X 076$ & abacbdecde & $-5.3405(74)$ & $-5.3407(31)$ & 0.0003 & $-5.3407(29)$ \\
\hline$X 077$ & abacbdeced & $3.5459(86)$ & $3.5604(51)$ & -0.0146 & $3.5567(43)$ \\
\hline$X 078$ & abacbdedce & $1.1666(80)$ & $1.1778(48)$ & -0.0112 & $1.1748(41)$ \\
\hline$X 079$ & abacbdedec & $5.3956(305)$ & $5.4128(205)$ & -0.0173 & $5.4075(170)$ \\
\hline$X 080$ & abacbdeecd & 0.4597 (257) & 0.4648 (217) & -0.0051 & $0.4627(166)$ \\
\hline$X 081$ & abacbdeedc & $-5.6566(248)$ & $-5.6298(217)$ & -0.0268 & $-5.6414(163)$ \\
\hline$X 082$ & abaccdbeed & $-8.5156(348)$ & $-8.4810(195)$ & -0.0345 & $-8.4893(170)$ \\
\hline$X 083$ & $a b a c c d d e e b$ & $18.7464(346)$ & $18.7522(207)$ & -0.0057 & 18.7507 (177) \\
\hline$X 084$ & abaccdebde & 8.9888 (129) & 8.9968 (209) & -0.0080 & $8.9911(110)$ \\
\hline$X 085$ & abaccdebed & $-2.2833(197)$ & $-2.2933(213)$ & 0.0100 & $-2.2879(144)$ \\
\hline$X 086$ & abaccdedbe & $0.5180(223)$ & $0.5162(218)$ & 0.0018 & $0.5171(155)$ \\
\hline$X 087$ & abaccdedeb & -16.5849 (349) & $-16.5942(173)$ & 0.0093 & $-16.5923(155)$ \\
\hline$X 088$ & abaccdeebd & $-5.2606(340)$ & $-5.2320(197)$ & -0.0286 & $-5.2392(171)$ \\
\hline$X 089$ & abaccdeedb & $12.6789(341)$ & 12.6723 (194) & 0.0066 & 12.6739 (169) \\
\hline$X 090$ & abacdbceed & $1.5206(130)$ & $1.5285(211)$ & -0.0079 & $1.5228(111)$ \\
\hline$X 091$ & abacdbdece & $-1.6355(97)$ & $-1.6320(58)$ & -0.0035 & $-1.6330(50)$ \\
\hline X092 & abacdbdeec & $2.1303(218)$ & $2.1083(201)$ & 0.0220 & 2.1184 (147) \\
\hline$X 093$ & abacdbecde & $-1.7594(42)$ & $-1.7538(16)$ & -0.0056 & $-1.7545(15)$ \\
\hline X094 & abacdbeced & $-1.0419(67)$ & $-1.0406(20)$ & -0.0014 & -1.0407 (19) \\
\hline$X 095$ & abacdbedce & $0.5838(35)$ & $0.5875(11)$ & -0.0037 & $0.5872(11)$ \\
\hline$X 096$ & abacdbedec & $1.3458(73)$ & $1.3495(22)$ & -0.0037 & $1.3492(21)$ \\
\hline X097 & abacdbeecd & $5.0319(89)$ & $5.0183(195)$ & 0.0136 & $5.0296(81)$ \\
\hline X098 & abacdbeedc & $-1.9806(183)$ & $-2.0218(215)$ & 0.0411 & -1.9979 (139) \\
\hline X099 & abacdcbeed & $3.0771(187)$ & $3.0553(218)$ & 0.0218 & $3.0678(142)$ \\
\hline$X 100$ & $a b a c d c d e e b$ & $-15.2919(331)$ & $-15.2360(203)$ & -0.0559 & $-15.2513(173)$ \\
\hline$X 101$ & abacdcebde & $-0.2462(64)$ & $-0.2397(26)$ & -0.0065 & $-0.2406(24)$ \\
\hline$X 102$ & abacdcebed & $-1.2883(75)$ & $-1.2953(34)$ & 0.0070 & $-1.2941(31)$ \\
\hline$X 103$ & abacdcedbe & $0.9424(74)$ & $0.9482(21)$ & -0.0057 & $0.9477(20)$ \\
\hline$X 104$ & $a b a c d c e d e b$ & $6.4131(298)$ & $6.3706(217)$ & 0.0426 & $6.3853(175)$ \\
\hline
\end{tabular}


TABLE I. (Continued)

\begin{tabular}{|c|c|c|c|c|c|}
\hline Diagram $\mathcal{G}$ & Expression & Value (Error) in Ref. [34] & Value (Error) in this work & Difference & Weighted average \\
\hline$X 105$ & abacdceebd & $3.0503(215)$ & $3.0491(216)$ & 0.0012 & $3.0497(152)$ \\
\hline$X 106$ & $a b a c d c e e d b$ & $-11.5662(344)$ & $-11.5384(201)$ & -0.0277 & $-11.5455(174)$ \\
\hline$X 107$ & abacddbeec & $-4.6573(345)$ & $-4.6265(193)$ & -0.0308 & $-4.6338(168)$ \\
\hline$X 108$ & $a b a c d d c e e b$ & $12.9775(341)$ & $12.9927(193)$ & -0.0152 & $12.9890(168)$ \\
\hline$X 109$ & abacddebce & $-0.0860(85)$ & $-0.0841(182)$ & -0.0019 & $-0.0857(77)$ \\
\hline$X 110$ & abacddebec & $1.9248(204)$ & $1.9014(204)$ & 0.0234 & $1.9131(144)$ \\
\hline$X 111$ & abacddecbe & $3.3578(132)$ & $3.3641(190)$ & -0.0062 & $3.3599(108)$ \\
\hline$X 112$ & $a b a c d d e c e b$ & $-11.8998(332)$ & $-11.8990(208)$ & -0.0008 & $-11.8992(176)$ \\
\hline$X 113$ & $a b a c d d e e b c$ & $-4.3847(322)$ & $-4.4412(176)$ & 0.0565 & $-4.4282(155)$ \\
\hline$X 114$ & $a b a c d d e e c b$ & $11.0641(343)$ & $11.0287(196)$ & 0.0355 & $11.0374(170)$ \\
\hline$X 115$ & abacdebcde & $-0.5974(52)$ & $-0.6028(20)$ & 0.0054 & $-0.6020(19)$ \\
\hline$X 116$ & abacdebced & $1.8362(28)$ & $1.8400(11)$ & -0.0038 & $1.8394(10)$ \\
\hline$X 117$ & abacdebdce & $0.3292(27)$ & $0.3309(10)$ & -0.0016 & 0.3307 (9) \\
\hline$X 118$ & abacdebdec & $-3.2721(55)$ & $-3.2764(16)$ & 0.0043 & $-3.2761(16)$ \\
\hline$X 119$ & abacdebecd & $-0.0751(53)$ & $-0.0716(18)$ & -0.0035 & $-0.0720(17)$ \\
\hline$X 120$ & abacdebedc & $1.8769(72)$ & $1.8847(22)$ & -0.0078 & $1.8840(21)$ \\
\hline$X 121$ & abacdecbde & $-0.8549(43)$ & $-0.8511(14)$ & -0.0039 & $-0.8515(13)$ \\
\hline$X 122$ & abacdecbed & $-0.7337(42)$ & $-0.7346(15)$ & 0.0008 & $-0.7345(14)$ \\
\hline$X 123$ & abacdecdbe & $-3.3559(67)$ & $-3.3564(21)$ & 0.0004 & $-3.3563(20)$ \\
\hline$X 124$ & $a b a c d e c d e b$ & $11.5746(106)$ & $11.5778(204)$ & -0.0033 & $11.5752(94)$ \\
\hline$X 125$ & abacdecebd & 0.8677 & $0.8710(19)$ & -0.0033 & 0.8707 (18) \\
\hline$X 126$ & abacdecedb & $-1.5696(162)$ & -1.5809 (199) & 0.0113 & $-1.5741(125)$ \\
\hline$X 127$ & abacdedbce & $1.1412(46)$ & 1.1495 (17) & -0.0083 & $1.1484(16)$ \\
\hline$X 128$ & abacdedbec & 0.6493 (59) & $0.6521(17)$ & -0.0027 & 0.6518 (16) \\
\hline$X 129$ & abacdedcbe & $1.4833(70)$ & $1.4890(21)$ & -0.0057 & $1.4885(20)$ \\
\hline$X 130$ & $a b a c d e d c e b$ & $-1.5696(180)$ & $-1.5797(205)$ & 0.0102 & $-1.5740(135)$ \\
\hline$X 131$ & $a b a c d e d e b c$ & 3.1060 (287) & 3.0832 (219) & 0.0228 & $3.0916(174)$ \\
\hline$X 132$ & abacdedecb & $-8.8300(337)$ & $-8.8562(198)$ & 0.0262 & $-8.8495(170)$ \\
\hline$X 133$ & abacdeebcd & $2.7263(88)$ & 2.7345 (177) & -0.0082 & 2.7279 (79) \\
\hline$X 134$ & $a b a c d e e b d c$ & $-0.6712(123)$ & -0.6569 (198) & -0.0143 & $-0.6672(104)$ \\
\hline$X 135$ & abacdeecbd & $0.9256(153)$ & $0.9201(207)$ & 0.0054 & $0.9236(123)$ \\
\hline$X 136$ & $a b a c d e e c d b$ & $-7.5256(305)$ & $-7.5147(205)$ & -0.0110 & $-7.5181(170)$ \\
\hline$X 137$ & $a b a c d e e d b c$ & $-2.3541(233)$ & $-2.3413(209)$ & -0.0128 & $-2.3470(156)$ \\
\hline$X 138$ & $a b a c d e e d c b$ & $10.1610(284)$ & $10.1624(215)$ & -0.0014 & $10.1619(171)$ \\
\hline$X 139$ & abbcaddeec & $14.8650(348)$ & $14.8877(203)$ & -0.0227 & 14.8819 (176) \\
\hline$X 140$ & abbcadeced & $-2.7901(206)$ & $-2.8044(207)$ & 0.0143 & $-2.7972(146)$ \\
\hline$X 141$ & abbcadedec & $-12.5567(350)$ & -12.4879 (207) & -0.0688 & $-12.5057(178)$ \\
\hline$X 142$ & abbcadeecd & $-1.5767(341)$ & $-1.5679(202)$ & -0.0088 & $-1.5702(173)$ \\
\hline$X 143$ & abbcadeedc & $10.3225(341)$ & 10.3377 (195) & -0.0152 & 10.3339 (169) \\
\hline$X 144$ & abbccdedea & $23.7239(368)$ & 23.6713 (189) & 0.0526 & $23.6823(168)$ \\
\hline$X 145$ & abbccdeeda & $-18.6212(349)$ & $-18.6295(166)$ & 0.0083 & $-18.6279(150)$ \\
\hline$X 146$ & abbcdadeec & -2.2990 & $-2.2458(202)$ & -0.0532 & $-2.2600(173)$ \\
\hline$X 147$ & abbcdaeced & $1.1243(55)$ & $1.1316(101)$ & -0.0074 & $1.1259(48)$ \\
\hline$X 148$ & abbcdaedec & $-1.4150(76)$ & $-1.4002(127)$ & -0.0148 & $-1.4111(65)$ \\
\hline$X 149$ & abbcdaeecd & $-8.3898(139)$ & $-8.3948(200)$ & 0.0050 & $-8.3914(114)$ \\
\hline$X 150$ & $a b b c d a e e d c$ & $2.8758(260)$ & $2.9171(200)$ & -0.0413 & 2.9017 (158) \\
\hline$X 151$ & abbcdcedea & $-10.9362(344)$ & -10.9329 (199) & -0.0033 & $-10.9337(172)$ \\
\hline$X 152$ & abbcdceeda & $14.6793(345)$ & $14.6367(201)$ & 0.0426 & 14.6475 (173) \\
\hline$X 153$ & abbcddecea & $14.8936(343)$ & 14.8523 (199) & 0.0414 & $14.8627(172)$ \\
\hline$X 154$ & abbcddeeca & $-20.6285(342)$ & $-20.5999(200)$ & -0.0286 & $-20.6072(173)$ \\
\hline$X 155$ & abbcdeadec & $5.0341(46)$ & $5.0371(75)$ & -0.0030 & $5.0349(39)$ \\
\hline$X 156$ & $a b b c d e a e d c$ & $-0.8277(69)$ & $-0.8285(130)$ & 0.0008 & $-0.8279(60)$ \\
\hline$X 157$ & abbcdecdea & $-11.8490(252)$ & $-11.8884(205)$ & 0.0394 & -11.8727 (159) \\
\hline$X 158$ & abbcdeceda & 0.4607 (329) & $0.4106(206)$ & 0.0502 & 0.4247 (174) \\
\hline$X 159$ & abbcdedcea & $0.4435(351)$ & $0.4270(173)$ & 0.0165 & $0.4302(155)$ \\
\hline
\end{tabular}


TABLE I. (Continued)

\begin{tabular}{|c|c|c|c|c|c|}
\hline Diagram $\mathcal{G}$ & Expression & Value (Error) in Ref. [34] & Value (Error) in this work & Difference & Weighted average \\
\hline$X 160$ & abbcdedeca & $14.0724(349)$ & $14.0370(197)$ & 0.0354 & $14.0455(171)$ \\
\hline$X 161$ & $a b b c d e e c d a$ & $7.8073(342)$ & $7.7941(198)$ & 0.0131 & $7.7974(171)$ \\
\hline$X 162$ & $a b b c d e e d c a$ & $-12.8293(339)$ & $-12.8564(195)$ & 0.0271 & $-12.8496(169)$ \\
\hline$X 163$ & abcabdceed & $6.8168(202)$ & $6.8111(214)$ & 0.0057 & $6.8141(147)$ \\
\hline$X 164$ & abcabddeec & $-12.8880(208)$ & $-12.8941(180)$ & 0.0061 & $-12.8915(136)$ \\
\hline$X 165$ & abcabdeced & $-2.1661(76)$ & $-2.1641(22)$ & -0.0020 & $-2.1643(21)$ \\
\hline$X 166$ & abcabdedce & $-2.3081(70)$ & $-2.3088(25)$ & 0.0008 & $-2.3087(23)$ \\
\hline$X 167$ & abcabdedec & $12.1361(150)$ & $12.1348(196)$ & 0.0013 & $12.1356(119)$ \\
\hline$X 168$ & abcabdeecd & $3.4447(120)$ & $3.4443(195)$ & 0.0004 & $3.4446(102)$ \\
\hline$X 169$ & abcabdeedc & $-6.9379(108)$ & $-6.9384(193)$ & 0.0005 & $-6.9380(94)$ \\
\hline$X 170$ & abcacdbeed & $0.2635(288)$ & 0.2420 & 0.0215 & $0.2482(154)$ \\
\hline$X 171$ & $a b c a c d d e e b$ & $-2.5229(313)$ & $-2.5628(194)$ & 0.0399 & $-2.5517(165)$ \\
\hline$X 172$ & abcacdebed & $1.5601(76)$ & 1.5697 (32) & -0.0096 & $1.5683(30)$ \\
\hline$X 173$ & $a b c a c d e d e b$ & $0.0193(298)$ & $-0.0209(215)$ & 0.0401 & $-0.0071(174)$ \\
\hline$X 174$ & abcacdeebd & $1.7158(191)$ & $1.7123(203)$ & 0.0035 & 1.7142 (139) \\
\hline$X 175$ & $a b c a c d e e d b$ & $-1.8253(175)$ & $-1.8346(206)$ & 0.0093 & $-1.8292(133)$ \\
\hline$X 176$ & abcadbceed & $0.7450(35)$ & $0.7430(64)$ & 0.0021 & $0.7446(30)$ \\
\hline$X 177$ & $a b c a d b d e e c$ & $0.0079(81)$ & 0.0411 (196) & -0.0332 & $0.0127(74)$ \\
\hline$X 178$ & abcadbeced & $0.7158(28)$ & $0.7230(9)$ & -0.0072 & 0.7223 \\
\hline$X 179$ & abcadbedce & -0.4377 (9) & -0.4380 & 0.0003 & -0.4379 \\
\hline$X 180$ & abcadbedec & $0.0284(25)$ & $0.0265(9)$ & 0.0020 & 0.0267 (9) \\
\hline$X 181$ & abcadbeecd & $-4.4372(28)$ & $-4.4261(61)$ & -0.0112 & $-4.4353(25)$ \\
\hline$X 182$ & $a b c a d b e e d c$ & $1.2822(43)$ & $1.2771(50)$ & 0.0051 & $1.2800(33)$ \\
\hline$X 183$ & abcadcbeed & $-0.0791(29)$ & $-0.0789(51)$ & -0.0001 & $-0.0790(25)$ \\
\hline$X 184$ & $a b c a d c d e e b$ & 0.1973 (134) & $0.2284(212)$ & -0.0311 & $0.2062(113)$ \\
\hline$X 185$ & abcadcebed & $-0.1269(16)$ & $-0.1264(9)$ & -0.0005 & $-0.1266(8)$ \\
\hline$X 186$ & $a b c a d c e d e b$ & $1.1883(21)$ & $1.1905(9)$ & -0.0022 & $1.1902(8)$ \\
\hline$X 187$ & abcadceebd & $1.2699(27)$ & $1.2700(43)$ & -0.0001 & $1.2699(23)$ \\
\hline$X 188$ & $a b c a d c e e d b$ & $1.7966(36)$ & $1.7937(72)$ & 0.0029 & $1.7960(32)$ \\
\hline$X 189$ & abcaddbeec & $-3.7500(105)$ & $-3.7574(175)$ & 0.0073 & $-3.7520(90)$ \\
\hline$X 190$ & $a b c a d d c e e b$ & $-2.4966(217)$ & $-2.4741(200)$ & -0.0225 & $-2.4845(147)$ \\
\hline$X 191$ & abcaddebec & $0.1892(62)$ & $0.1892(69)$ & 0.0001 & $0.1892(46)$ \\
\hline$X 192$ & $a b c a d d e c e b$ & $2.3868(91)$ & $2.3870(180)$ & -0.0003 & $2.3868(81)$ \\
\hline$X 193$ & $a b c a d d e e b c$ & $-4.2570(84)$ & $-4.2686(128)$ & 0.0116 & $-4.2605(70)$ \\
\hline$X 194$ & $a b c a d d e e c b$ & $-0.6785(102)$ & $-0.6797(188)$ & 0.0012 & $-0.6787(89)$ \\
\hline$X 195$ & abcadebcde & $-1.0708(20)$ & $-1.0706(9)$ & -0.0002 & $-1.0706(8)$ \\
\hline$X 196$ & abcadebced & $-2.0432(20)$ & $-2.0473(9)$ & 0.0040 & $-2.0466(8)$ \\
\hline$X 197$ & abcadebdce & -0.3848 & -0.3838 & -0.0010 & -0.3840 \\
\hline$X 198$ & abcadebdec & $-2.3533(26)$ & $-2.3583(9)$ & 0.0050 & $-2.3577(8)$ \\
\hline$X 199$ & abcadebecd & $1.0636(26)$ & 1.0667 (9) & -0.0031 & $1.0664(8)$ \\
\hline$X 200$ & abcadebedc & $0.0266(26)$ & $0.0259(9)$ & 0.0007 & $0.0260(9)$ \\
\hline$X 201$ & abcadecbde & $-0.4897(18)$ & $-0.4887(8)$ & -0.0010 & -0.4889 \\
\hline$X 202$ & abcadecbed & $1.9313(17)$ & $1.9363(9)$ & -0.0050 & $1.9352(8)$ \\
\hline$X 203$ & abcadecdbe & $0.9061(10)$ & $0.9075(7)$ & -0.0014 & $0.9071(5)$ \\
\hline$X 204$ & $a b c a d e c d e b$ & $-1.9485(26)$ & -1.9449 (9) & -0.0036 & $-1.9453(8)$ \\
\hline$X 205$ & abcadecebd & $-0.9039(13)$ & $-0.9044(8)$ & 0.0005 & $-0.9043(7)$ \\
\hline$X 206$ & $a b c a d e c e d b$ & $1.6836(23)$ & $1.6829(9)$ & 0.0007 & $1.6830(8)$ \\
\hline$X 207$ & abcadedbce & $0.2908(23)$ & $0.2959(9)$ & -0.0051 & $0.2952(8)$ \\
\hline$X 208$ & abcadedbec & $0.5283(28)$ & $0.5312(9)$ & -0.0028 & $0.5309(8)$ \\
\hline$X 209$ & abcadedcbe & 0.1496 (19) & $0.1511(8)$ & -0.0015 & $0.1509(8)$ \\
\hline$X 210$ & $a b c a d e d c e b$ & 0.7803 (19) & 0.7800 (9) & 0.0003 & $0.7801(8)$ \\
\hline$X 211$ & $a b c a d e d e b c$ & $5.1339(90)$ & $5.1463(129)$ & -0.0124 & $5.1379(74)$ \\
\hline$X 212$ & $a b c a d e d e c b$ & $-0.4617(138)$ & $-0.4539(206)$ & -0.0078 & $-0.4593(114)$ \\
\hline$X 213$ & abcadeebcd & $-2.4516(29)$ & $-2.4515(42)$ & -0.0001 & $-2.4516(23)$ \\
\hline$X 214$ & $a b c a d e e b d c$ & 0.6801 (39) & 0.6777 (69) & 0.0023 & $0.6795(34)$ \\
\hline
\end{tabular}


TABLE I. (Continued)

\begin{tabular}{|c|c|c|c|c|c|}
\hline Diagram $\mathcal{G}$ & Expression & Value (Error) in Ref. [34] & Value (Error) in this work & Difference & Weighted average \\
\hline$X 215$ & abcadeecbd & $0.0724(24)$ & $0.0763(43)$ & -0.0038 & $0.0734(21)$ \\
\hline$X 216$ & abcadeecdb & $-1.3029(42)$ & $-1.3013(56)$ & -0.0016 & $-1.3023(33)$ \\
\hline$X 217$ & abcadeedbc & $-2.2261(71)$ & $-2.2348(117)$ & 0.0086 & $-2.2285(60)$ \\
\hline$X 218$ & $a b c a d e e d c b$ & $-1.6396(84)$ & $-1.6319(92)$ & -0.0077 & $-1.6361(62)$ \\
\hline$X 219$ & abcbaddeec & $1.3579(311)$ & $1.3595(178)$ & -0.0015 & $1.3591(155)$ \\
\hline$X 220$ & abcbadedec & $-2.5734(222)$ & $-2.5667(214)$ & -0.0068 & $-2.5699(154)$ \\
\hline$X 221$ & abcbadeecd & $0.6650(161)$ & $0.6680(206)$ & -0.0030 & $0.6662(127)$ \\
\hline$X 222$ & abcbadeedc & $0.8293(178)$ & $0.8025(201)$ & 0.0267 & $0.8175(133)$ \\
\hline$X 223$ & $a b c b c d e e d a$ & $17.5168(349)$ & $17.5020(156)$ & 0.0148 & $17.5045(142)$ \\
\hline$X 224$ & abcbdadeec & $2.4729(110)$ & $2.5001(196)$ & -0.0273 & $2.4794(96)$ \\
\hline$X 225$ & abcbdaedec & $0.3434(39)$ & 0.3419 (12) & 0.0015 & $0.3421(12)$ \\
\hline$X 226$ & abcbdaeecd & $1.0443(58)$ & $1.0390(77)$ & 0.0052 & $1.0424(46)$ \\
\hline$X 227$ & abcbdaeedc & $0.5835(97)$ & $0.5991(203)$ & -0.0156 & $0.5864(87)$ \\
\hline$X 228$ & abcbdceeda & $-6.8113(333)$ & $-6.8120(208)$ & 0.0007 & $-6.8118(176)$ \\
\hline$X 229$ & abcbddaeec & $-1.9843(323)$ & $-1.9807(210)$ & -0.0036 & $-1.9818(176)$ \\
\hline$X 230$ & abcbddeeca & $15.6844(350)$ & 15.6718 (199) & 0.0126 & 15.6749 (173) \\
\hline$X 231$ & abcbdeadec & $-0.7737(28)$ & $-0.7723(10)$ & -0.0014 & $-0.7725(10)$ \\
\hline$X 232$ & abcbdeaedc & 0.4608 & 0.4604 (12) & 0.0004 & 0.4605 (11) \\
\hline$X 233$ & abcbdecdea & $8.6698(116)$ & 8.6613 (192) & 0.0085 & 8.6675 (99) \\
\hline$X 234$ & abcbdeceda & $-2.5793(179)$ & $-2.5995(210)$ & 0.0202 & $-2.5878(136)$ \\
\hline$X 235$ & abcbdedaec & $0.7486(35)$ & $0.7478(11)$ & 0.0009 & $0.7478(10)$ \\
\hline$X 236$ & abcbdedcea & $2.0560(180)$ & $2.1072(205)$ & -0.0512 & $2.0782(135)$ \\
\hline$X 237$ & abcbdedeca & $-12.9913(363)$ & $-12.9686(187)$ & -0.0227 & $-12.9734(166)$ \\
\hline$X 238$ & $a b c b d e e a d c$ & $1.2747(45)$ & $1.2837(92)$ & -0.0090 & $1.2765(41)$ \\
\hline$X 239$ & abcbdeecda & $-2.8075(345)$ & $-2.8021(201)$ & -0.0053 & $-2.8035(174)$ \\
\hline$X 240$ & abcbdeedca & $10.9428(298)$ & 10.9241 (209) & 0.0187 & $10.9303(171)$ \\
\hline$X 241$ & $a b c c a d d e e b$ & $13.8142(357)$ & $13.7745(201)$ & 0.0397 & $13.7841(175)$ \\
\hline$X 242$ & $a b c c a d e d e b$ & $-10.4867(377)$ & -10.4478 (177) & -0.0389 & $-10.4549(160)$ \\
\hline$X 243$ & $a b c c a d e e d b$ & $3.8891(336)$ & 3.8802 (199) & 0.0089 & 3.8825 (171) \\
\hline$X 244$ & $a b c c d a d e e b$ & $-3.3041(334)$ & $-3.2721(187)$ & -0.0320 & $-3.2797(163)$ \\
\hline$X 245$ & $a b c c d a e d e b$ & $0.0658(83)$ & $0.0880(192)$ & -0.0222 & $0.0693(76)$ \\
\hline$X 246$ & $a b c c d a e e d b$ & $-0.3959(174)$ & $-0.3816(213)$ & -0.0143 & $-0.3902(134)$ \\
\hline$X 247$ & $a b c c d d a e e b$ & $15.9539(344)$ & $15.9573(191)$ & -0.0034 & 15.9565 (167) \\
\hline$X 248$ & $a b c c d d e a e b$ & $-1.9165(278)$ & $-1.9008(209)$ & -0.0157 & $-1.9065(167)$ \\
\hline$X 249$ & $a b c c d e a d e b$ & $4.0116(46)$ & $4.0143(66)$ & -0.0027 & $4.0125(37)$ \\
\hline$X 250$ & $a b c c d e a e d b$ & $-1.0558(68)$ & $-1.0478(128)$ & -0.0080 & $-1.0540(60)$ \\
\hline$X 251$ & $a b c c d e d a e b$ & $-1.3906(76)$ & $-1.3435(198)$ & -0.0472 & $-1.3846(71)$ \\
\hline$X 252$ & $a b c c d e d e a b$ & $-10.9091(343)$ & -10.8565 (179) & -0.0526 & -10.8677 (158) \\
\hline$X 253$ & abccdedeba & $17.8437(352)$ & $17.8230(196)$ & 0.0207 & $17.8279(171)$ \\
\hline$X 254$ & $a b c c d e e a d b$ & $2.2265(175)$ & $2.2133(217)$ & 0.0132 & $2.2213(136)$ \\
\hline$X 255$ & $a b c c d e e d a b$ & $8.1598(340)$ & $8.1520(173)$ & 0.0078 & $8.1536(154)$ \\
\hline$X 256$ & $a b c c d e e d b a$ & $-14.0405(342)$ & -13.9856 (194) & -0.0549 & -13.9990 (169) \\
\hline$X 257$ & abcdabceed & $5.7475(51)$ & $5.7447(79)$ & 0.0029 & $5.7467(43)$ \\
\hline$X 258$ & $a b c d a b d e e c$ & $-0.5254(39)$ & $-0.5246(55)$ & -0.0008 & $-0.5252(32)$ \\
\hline$X 259$ & abcdabeced & $0.0053(27)$ & 0.0050 (10) & 0.0003 & $0.0050(9)$ \\
\hline$X 260$ & abcdabedec & $-0.3958(20)$ & $-0.3927(8)$ & -0.0031 & $-0.3932(8)$ \\
\hline$X 261$ & abcdabeecd & $6.4046(30)$ & $6.3974(50)$ & 0.0072 & $6.4027(26)$ \\
\hline$X 262$ & abcdabeedc & $-2.2854(24)$ & -2.2848 & -0.0005 & $-2.2852(20)$ \\
\hline$X 263$ & abcdacbeed & $-2.8330(35)$ & $-2.8190(62)$ & -0.0139 & $-2.8297(30)$ \\
\hline$X 264$ & $a b c d a c d e e b$ & $4.8826(64)$ & $4.8752(95)$ & 0.0074 & $4.8803(53)$ \\
\hline$X 265$ & abcdacebed & $-0.6756(20)$ & -0.6730 & -0.0026 & $-0.6734(8)$ \\
\hline$X 266$ & $a b c d a c e d e b$ & $0.1206(23)$ & 0.1225 (9) & -0.0019 & $0.1223(8)$ \\
\hline$X 267$ & abcdaceebd & -0.6608 (19) & $-0.6591(31)$ & -0.0017 & $-0.6603(16)$ \\
\hline$X 268$ & $a b c d a c e e d b$ & $0.1185(31)$ & $0.1214(56)$ & -0.0029 & 0.1192 (27) \\
\hline$X 269$ & abcdadbeec & $-0.7190(56)$ & $-0.7206(88)$ & 0.0016 & $-0.7195(47)$ \\
\hline
\end{tabular}


TABLE I. (Continued)

\begin{tabular}{|c|c|c|c|c|c|}
\hline Diagram $\mathcal{G}$ & Expression & Value (Error) in Ref. [34] & Value (Error) in this work & Difference & Weighted average \\
\hline$X 270$ & $a b c d a d c e e b$ & $-1.6881(97)$ & $-1.6705(217)$ & -0.0176 & $-1.6851(88)$ \\
\hline$X 271$ & abcdadebec & $0.2492(23)$ & $0.2505(9)$ & -0.0012 & $0.2503(8)$ \\
\hline$X 272$ & $a b c d a d e c e b$ & $-0.7285(32)$ & $-0.7297(11)$ & 0.0012 & $-0.7296(10)$ \\
\hline$X 273$ & $a b c d a d e e b c$ & $-2.0474(45)$ & $-2.0422(75)$ & -0.0052 & $-2.0460(39)$ \\
\hline$X 274$ & $a b c d a d e e c b$ & $0.8675(72)$ & $0.8768(117)$ & -0.0093 & $0.8701(61)$ \\
\hline$X 275$ & abcdaebced & $-0.7496(12)$ & $-0.7478(8)$ & -0.0018 & $-0.7484(7)$ \\
\hline$X 276$ & abcdaebdce & $-0.5547(10)$ & $-0.5540(5)$ & -0.0007 & $-0.5541(4)$ \\
\hline$X 277$ & abcdaebdec & $2.7936(10)$ & $2.7944(6)$ & -0.0008 & $2.7942(5)$ \\
\hline$X 278$ & abcdaebecd & $-0.1577(23)$ & $-0.1602(9)$ & 0.0025 & $-0.1598(9)$ \\
\hline$X 279$ & abcdaebedc & $0.8399(15)$ & $0.8408(8)$ & -0.0009 & $0.8406(7)$ \\
\hline$X 280$ & abcdaecbed & $-1.0127(8)$ & $-1.0114(5)$ & -0.0013 & -1.0118 \\
\hline$X 281$ & $a b c d a e c d e b$ & $-1.3732(25)$ & $-1.3703(9)$ & -0.0030 & -1.3707 (9) \\
\hline$X 282$ & abcdaecebd & 0.4907 (18) & $0.4922(9)$ & -0.0015 & $0.4919(8)$ \\
\hline$X 283$ & $a b c d a e c e d b$ & $-0.0427(23)$ & $-0.0396(8)$ & -0.0030 & $-0.0400(8)$ \\
\hline$X 284$ & abcdaedbec & $-0.2670(9)$ & $-0.2673(5)$ & 0.0003 & -0.2673 \\
\hline$X 285$ & $a b c d a e d c e b$ & $0.0271(16)$ & 0.0277 (7) & -0.0007 & $0.0276(6)$ \\
\hline$X 286$ & $a b c d a e d e b c$ & $0.8014(21)$ & $0.8041(9)$ & -0.0026 & $0.8036(8)$ \\
\hline$X 287$ & $a b c d a e d e c b$ & $0.2014(19)$ & $0.2021(9)$ & -0.0008 & $0.2020(8)$ \\
\hline$X 288$ & abcdaeebcd & $4.2112(28)$ & $4.2137(45)$ & -0.0025 & $4.2119(24)$ \\
\hline$X 289$ & $a b c d a e e b d c$ & $-1.5651(19)$ & $-1.5662(35)$ & 0.0011 & $-1.5654(17)$ \\
\hline$X 290$ & abcdaeecbd & $-3.7763(23)$ & $-3.7736(42)$ & -0.0027 & $-3.7756(20)$ \\
\hline$X 291$ & $a b c d a e e c d b$ & $1.5957(32)$ & $1.5890(62)$ & 0.0067 & $1.5943(28)$ \\
\hline$X 292$ & $a b c d a e e d b c$ & $0.9114(36)$ & 0.9144 (49) & -0.0030 & $0.9125(29)$ \\
\hline$X 293$ & $a b c d a e e d c b$ & $-1.2653(41)$ & $-1.2582(58)$ & -0.0070 & $-1.2629(33)$ \\
\hline$X 294$ & abcdbaceed & $-3.3891(25)$ & $-3.3873(36)$ & -0.0018 & $-3.3885(20)$ \\
\hline$X 295$ & $a b c d b a d e e c$ & $1.7883(26)$ & 1.7913 (44) & -0.0030 & $1.7891(22)$ \\
\hline$X 296$ & abcdbaeced & $0.5511(13)$ & $0.5528(8)$ & -0.0017 & $0.5522(7)$ \\
\hline$X 297$ & abcdbaedec & $-0.4696(16)$ & -0.4693 & -0.0003 & $-0.4694(7)$ \\
\hline$X 298$ & abcdbaeecd & $-1.9142(28)$ & $-1.9153(44)$ & 0.0011 & $-1.9145(23)$ \\
\hline$X 299$ & $a b c d b a e e d c$ & $-0.2907(22)$ & -0.2887 (39) & -0.0020 & -0.2902 (19) \\
\hline$X 300$ & $a b c d b c e e d a$ & $-9.4327(194)$ & $-9.4309(210)$ & -0.0018 & $-9.4318(142)$ \\
\hline$X 301$ & $a b c d b d a e e c$ & $-1.3351(81)$ & $-1.3445(122)$ & 0.0094 & $-1.3380(68)$ \\
\hline$X 302$ & $a b c d b d e e c a$ & $-1.8294(223)$ & $-1.8502(216)$ & 0.0208 & $-1.8401(155)$ \\
\hline$X 303$ & abcdbeadec & $0.3341(7)$ & 0.3348 & -0.0007 & 0.3347 \\
\hline$X 304$ & abcdbeaecd & $-0.3397(16)$ & $-0.3381(9)$ & -0.0016 & $-0.3385(8)$ \\
\hline$X 305$ & $a b c d b e a e d c$ & 0.4715 (14) & $0.4719(7)$ & -0.0004 & $0.4718(6)$ \\
\hline$X 306$ & abcdbeceda & $0.1228(55)$ & $0.1167(86)$ & 0.0062 & $0.1210(46)$ \\
\hline$X 307$ & abcdbedeca & $-0.3071(59)$ & $-0.3024(107)$ & -0.0048 & $-0.3060(52)$ \\
\hline$X 308$ & abcdbeeadc & $1.8122(22)$ & $1.8126(40)$ & -0.0004 & 1.8123 (19) \\
\hline$X 309$ & abcdbeecda & $-4.2448(173)$ & $-4.2500(213)$ & 0.0051 & -4.2469 (134) \\
\hline$X 310$ & abcdbeedca & 0.2490 (191) & $0.2397(211)$ & 0.0093 & $0.2448(142)$ \\
\hline$X 311$ & abcdcabeed & $-0.5291(58)$ & $-0.5389(78)$ & 0.0098 & $-0.5326(47)$ \\
\hline$X 312$ & $a b c d c a d e e b$ & $-1.2454(139)$ & $-1.2693(90)$ & 0.0239 & $-1.2622(76)$ \\
\hline$X 313$ & abcdcaebed & $0.9660(38)$ & 0.9654 (12) & 0.0006 & $0.9654(11)$ \\
\hline$X 314$ & $a b c d c a e d e b$ & $0.8266(29)$ & 0.8335 (11) & -0.0069 & $0.8327(10)$ \\
\hline$X 315$ & abcdcaeebd & $-1.3728(43)$ & $-1.3787(67)$ & 0.0059 & $-1.3745(36)$ \\
\hline$X 316$ & $a b c d c a e e d b$ & $0.0094(39)$ & $0.0272(89)$ & -0.0178 & $0.0123(36)$ \\
\hline$X 317$ & abcdcbeeda & $1.4535(221)$ & $1.4828(204)$ & -0.0293 & $1.4693(150)$ \\
\hline$X 318$ & $a b c d c d a e e b$ & $-8.7568(343)$ & $-8.7479(201)$ & -0.0089 & $-8.7502(174)$ \\
\hline$X 319$ & $a b c d c d e a e b$ & $0.6801(179)$ & $0.6449(213)$ & 0.0352 & $0.6655(137)$ \\
\hline$X 320$ & $a b c d c e a d e b$ & $0.5627(17)$ & $0.5641(9)$ & -0.0014 & $0.5637(8)$ \\
\hline$X 321$ & $a b c d c e a e d b$ & $-0.9005(26)$ & $-0.8961(10)$ & -0.0044 & $-0.8967(9)$ \\
\hline$X 322$ & $a b c d c e d a e b$ & 0.9338 (23) & $0.9364(9)$ & -0.0025 & $0.9360(9)$ \\
\hline$X 323$ & $a b c d c e e a d b$ & $-0.0053(40)$ & $0.0092(85)$ & -0.0145 & $-0.0026(36)$ \\
\hline$X 324$ & $a b c d c e e d a b$ & $-8.8058(243)$ & -8.8139 (209) & 0.0081 & $-8.8105(158)$ \\
\hline
\end{tabular}


TABLE I. (Continued)

\begin{tabular}{|c|c|c|c|c|c|}
\hline Diagram $\mathcal{G}$ & Expression & Value (Error) in Ref. [34] & Value (Error) in this work & Difference & Weighted average \\
\hline$X 325$ & $a b c d c e e d b a$ & $11.5958(343)$ & $11.5456(198)$ & 0.0503 & $11.5582(172)$ \\
\hline$X 326$ & abcddabeec & $-9.0047(145)$ & $-8.9830(204)$ & -0.0217 & $-8.9974(118)$ \\
\hline$X 327$ & $a b c d d a c e e b$ & $1.5517(229)$ & $1.5868(209)$ & -0.0351 & $1.5709(154)$ \\
\hline$X 328$ & abcddaebec & $-0.2781(42)$ & $-0.2745(74)$ & -0.0035 & $-0.2772(36)$ \\
\hline$X 329$ & $a b c d d a e c e b$ & $-0.9627(67)$ & $-0.9506(103)$ & -0.0121 & $-0.9591(56)$ \\
\hline$X 330$ & $a b c d d a e e b c$ & $-4.9591(88)$ & $-4.9451(125)$ & -0.0141 & $-4.9545(72)$ \\
\hline$X 331$ & $a b c d d a e e c b$ & $4.7241(127)$ & $4.7164(215)$ & 0.0077 & $4.7221(109)$ \\
\hline$X 332$ & $a b c d d b a e e c$ & $3.0539(161)$ & $3.0436(206)$ & 0.0103 & $3.0500(127)$ \\
\hline$X 333$ & abcddbeeca & $6.8088(341)$ & $6.8339(194)$ & -0.0251 & $6.8278(169)$ \\
\hline$X 334$ & $a b c d d c a e e b$ & $5.1727(340)$ & $5.1696(189)$ & 0.0031 & $5.1703(165)$ \\
\hline$X 335$ & $a b c d d c e a e b$ & $-2.0294(132)$ & $-2.0421(212)$ & 0.0127 & $-2.0329(112)$ \\
\hline$X 336$ & abcddeabec & $-0.7685(20)$ & -0.7730 & 0.0045 & $-0.7700(17)$ \\
\hline$X 337$ & $a b c d d e a c e b$ & $-1.2039(32)$ & $-1.1991(55)$ & -0.0048 & $-1.2027(27)$ \\
\hline$X 338$ & $a b c d d e a e b c$ & $-1.8505(38)$ & $-1.8492(69)$ & -0.0012 & $-1.8502(33)$ \\
\hline$X 339$ & $a b c d d e a e c b$ & $0.4111(40)$ & $0.4151(59)$ & -0.0040 & $0.4124(33)$ \\
\hline$X 340$ & abcddebeca & $-2.1543(202)$ & $-2.1472(214)$ & -0.0071 & $-2.1509(147)$ \\
\hline$X 341$ & $a b c d d e c a e b$ & $1.7815(33)$ & $1.7782(65)$ & 0.0033 & $1.7808(30)$ \\
\hline$X 342$ & $a b c d d e e a c b$ & $2.6063(125)$ & $2.6128(115)$ & -0.0065 & $2.6099(84)$ \\
\hline$X 343$ & abcdeabced & $3.8873(30)$ & $3.8962(10)$ & -0.0089 & $3.8952(9)$ \\
\hline$X 344$ & abcdeabdce & $3.4223(18)$ & $3.4239(9)$ & -0.0016 & $3.4236(8)$ \\
\hline$X 345$ & abcdeabdec & $-1.0075(18)$ & $-1.0069(9)$ & -0.0006 & $-1.0070(8)$ \\
\hline$X 346$ & abcdeabecd & 0.2864 (20) & $0.2904(9)$ & -0.0041 & $0.2898(8)$ \\
\hline$X 347$ & abcdeabedc & $-2.6846(21)$ & $-2.6875(9)$ & 0.0029 & $-2.6870(9)$ \\
\hline$X 348$ & abcdeacbed & $-0.4899(15)$ & -0.4905 (9) & 0.0005 & $-0.4903(7)$ \\
\hline$X 349$ & $a b c d e a c d e b$ & $2.0800(36)$ & $2.0793(9)$ & 0.0007 & $2.0794(9)$ \\
\hline$X 350$ & abcdeacebd & $1.4643(11)$ & $1.4649(6)$ & -0.0007 & $1.4648(5)$ \\
\hline$X 351$ & $a b c d e a c e d b$ & $0.2554(20)$ & $0.2536(8)$ & 0.0017 & $0.2539(8)$ \\
\hline$X 352$ & abcdeadbec & $-0.1260(8)$ & -0.1257 & -0.0003 & -0.1258 \\
\hline$X 353$ & $a b c d e a d c e b$ & $0.1950(16)$ & $0.1952(8)$ & -0.0002 & $0.1952(7)$ \\
\hline$X 354$ & $a b c d e a d e b c$ & $-2.0503(20)$ & $-2.0501(9)$ & -0.0002 & $-2.0501(8)$ \\
\hline$X 355$ & $a b c d e a d e c b$ & $-1.0738(25)$ & $-1.0747(9)$ & 0.0009 & $-1.0746(8)$ \\
\hline$X 356$ & abcdeaebcd & $2.0684(24)$ & $2.0685(9)$ & -0.0002 & $2.0685(9)$ \\
\hline$X 357$ & $a b c d e a e b d c$ & $0.3746(16)$ & $0.3760(8)$ & -0.0014 & $0.3757(7)$ \\
\hline$X 358$ & abcdeaecbd & $0.0463(16)$ & $0.0474(8)$ & -0.0011 & $0.0472(7)$ \\
\hline$X 359$ & $a b c d e a e c d b$ & $-0.1396(17)$ & -0.1381 & -0.0015 & $-0.1384(8)$ \\
\hline$X 360$ & $a b c d e a e d b c$ & $-0.4604(37)$ & $-0.4592(10)$ & -0.0012 & $-0.4593(10)$ \\
\hline$X 361$ & $a b c d e a e d c b$ & $2.5600(26)$ & $2.5629(9)$ & -0.0029 & $2.5625(8)$ \\
\hline$X 362$ & abcdebadec & $-0.5714(12)$ & -0.5729 & 0.0014 & $-0.5724(6)$ \\
\hline$X 363$ & abcdebaecd & $-2.3442(19)$ & $-2.3475(9)$ & 0.0033 & $-2.3468(8)$ \\
\hline$X 364$ & $a b c d e b a e d c$ & 2.3957 (18) & $2.4006(9)$ & -0.0049 & $2.3995(8)$ \\
\hline$X 365$ & abcdebceda & $0.4177(30)$ & $0.4187(44)$ & -0.0011 & $0.4180(24)$ \\
\hline$X 366$ & abcdebdeca & $5.6759(43)$ & $5.6790(110)$ & -0.0031 & $5.6763(40)$ \\
\hline$X 367$ & abcdebeadc & $-0.7176(12)$ & -0.7168 & -0.0008 & $-0.7170(7)$ \\
\hline$X 368$ & abcdebecda & $-0.3404(45)$ & $-0.3420(79)$ & 0.0015 & $-0.3408(39)$ \\
\hline$X 369$ & abcdebedca & $-3.3812(59)$ & $-3.3665(121)$ & -0.0147 & $-3.3783(53)$ \\
\hline$X 370$ & $a b c d e c a d e b$ & $-1.4763(12)$ & $-1.4741(7)$ & -0.0022 & $-1.4747(6)$ \\
\hline$X 371$ & $a b c d e c a e d b$ & $0.0045(10)$ & 0.0050 & -0.0005 & 0.0049 \\
\hline$X 372$ & $a b c d e c d a e b$ & $-1.2900(33)$ & $-1.2913(9)$ & 0.0013 & $-1.2912(9)$ \\
\hline$X 373$ & $a b c d e c e a d b$ & $0.5851(24)$ & 0.5877 (9) & -0.0025 & $0.5874(8)$ \\
\hline$X 374$ & $a b c d e c e d a b$ & $0.9188(266)$ & $0.9318(166)$ & -0.0130 & $0.9281(141)$ \\
\hline$X 375$ & $a b c d e c e d b a$ & $1.0991(163)$ & $1.0880(210)$ & 0.0111 & $1.0949(129)$ \\
\hline$X 376$ & abcdedabec & $1.0484(16)$ & $1.0514(7)$ & -0.0030 & $1.0509(6)$ \\
\hline X377 & $a b c d e d a c e b$ & $0.4264(27)$ & $0.4313(9)$ & -0.0049 & $0.4307(8)$ \\
\hline$X 378$ & $a b c d e d a e b c$ & $1.3196(21)$ & $1.3238(9)$ & -0.0042 & $1.3232(8)$ \\
\hline$X 379$ & $a b c d e d a e c b$ & $-0.3201(17)$ & -0.3198 & -0.0003 & $-0.3198(8)$ \\
\hline
\end{tabular}


TABLE I. (Continued)

\begin{tabular}{llcrrr}
\hline \hline Diagram $\mathcal{G}$ & Expression & Value (Error) in Ref. [34] & Value (Error) in this work & Difference & Weighted average \\
\hline X380 & abcdedbeca & $-1.0268(48)$ & $-1.0216(91)$ & -0.0052 & $-1.0257(43)$ \\
X381 & abcdedcaeb & $1.0861(29)$ & $1.0882(9)$ & -0.0022 & $1.0880(9)$ \\
X382 & abcdedeacb & $-1.7712(80)$ & $-1.7582(142)$ & -0.0130 & $-1.7681(70)$ \\
X383 & abcdeeabdc & $-4.8034(22)$ & $-4.7978(35)$ & -0.0056 & $-4.8018(19)$ \\
X384 & abcdeeacdb & $1.9266(31)$ & $1.9384(57)$ & -0.0118 & $1.9293(27)$ \\
X385 & abcdeeadbc & $-0.7427(19)$ & $-0.7408(30)$ & -0.0019 & $-0.7421(16)$ \\
X386 & abcdeeadcb & $0.6887(38)$ & $0.6877(59)$ & 0.0010 & $0.6884(32)$ \\
X387 & abcdeebdca & $1.9508(152)$ & $1.9763(208)$ & -0.0255 & $1.9597(123)$ \\
X388 & abcdeecadb & $-0.4349(40)$ & $-0.4336(49)$ & -0.0013 & $-0.4344(31)$ \\
X389 & abcdeedacb & $-0.0433(68)$ & $-0.0525(123)$ & 0.0092 & $-0.0455(59)$ \\
\hline \hline
\end{tabular}

HOKUSAI-BigWaterfall, which is almost twice faster than other two systems and allows us to increase the number of sampling points to $1.6 \times 10^{10}$. So far approximately $3.3 \times 10^{7}$ core $\cdot$ hours of the computer resources have been dedicated to obtain the 389 new values listed in Table I. All 389 integrals of new calculation have achieved the required precision and every uncertainties have been reduced to less than 0.022. After consistency between new and old calculations were checked for each integral, we combined two results except for the X024 integral. Finally, for every integral, the combined uncertainty is reduced to less than 0.018 .

\section{MAPPING OF FEYNMAN PARAMETERS}

The convergence speed of the VEGAS integration of a Set $\mathrm{V}$ integral does depend on a choice of mapping of the Feynman parameters to the integration variables, although analytically any choices of mappings should give the same value. The shape of an integrand looks very different if a mapping is different. A mapping should be chosen so that the shape of an integrand is as flat as possible in the entire integration domain. In addition, the results of VEGAS numerical integration with different mappings can be regarded as independent of each other.

In general, an integrand of the higher order perturbation theory of the anomalous magnetic moment has very sharp peaks where UV or IR cancellation occurs. Thus, a mapping must be selected in such a way that as small a number of sharp peaks as possible are formed as seen by the VEGAS integration variables. There is no definite rule to select the best mapping, but an integral shows better behavior and faster convergence against the VEGAS iterations if its mapping reflects the structure of divergent subdiagrams. The mappings of the previous calculation in Ref. [34] and also in this work were chosen according to this policy. When a mapping is properly chosen, singular regions are concentrated at the edges of only a few integration variables. This means that the sharp peaks spread out in the surface volumes of the whole integration domain. We further apply the power-law stretches to these edges before performing numerical integration in order to accelerate convergence [51].
For the new calculation we have rewritten the mappings of all 389 integrals to different but "better" ones. Recalculation of the Set V integrals with different mappings provides us with a useful check of reliability of the VEGAS integration results. Since the choice of mapping is not deterministic, it was not automated in the earlier version of GENCODEN, and a default thirteen-dimensional mapping was commonly used for all integrals of Set V. (The latest version of GENCODE $N$ writes down a mapping according to given instructions.) The mapping part occupies 30 lines of a FORTRAN code within about 100,000 lines of the integrand and it is the only part modified by the human hand before the numerical integration is carried out.

Even though the execution time is limited, the consistent results of the numerical integration with two different "better" mappings indicate that the VEGAS integration result should be reliable.

\section{MAPPING ERROR IN X024 CORRECTED}

We have integrated all 389 integrals of Set V according to the scheme outlined above. A preliminary result was reported in Ref. [34]. In order to improve it further we have carried out a new and independent numerical evaluation of the integrals of Set V. During this work we discovered that one of the integrals, called $X 024$, was given a wrong value in the previous calculation due to an incorrect mapping of Feynman parameters onto the VEGAS integration variables. The 14 Feynman parameters of $X 024$ subject to a linear constraint can be mapped onto an 11-dimensional unit hypercube because of the presence of two self-energy subdiagrams.

The $X 024$ has a vertex subdiagram $S_{78}$ consisting of the fermion lines 7 and 8 and the photon line $e$. It causes UV divergence when the sum $z_{78 e}=z_{7}+z_{8}+z_{e}$ tends to zero. Though its divergence is canceled by a UV counterterm, the integrand shows a very sharp peak near $z_{78 e}=0$. So, we assigned one of the VEGAS integration variables $q(i), i=1,2, \ldots$, to the sum $z_{78 e}$ as $z_{78 e} \propto q(9)$ that was further mapped as $z_{78}=z_{78 e} \times q(10)$ and $z_{e}=z_{78 e}-z_{78}$. We were supposed to divide $z_{78}$ into $z_{7}$ and $z_{8}$ using the eleventh integration variable such that $z_{7}=z_{78} \times q(11)$ 
and $z_{8}=z_{78}-z_{7}$. Instead, we treated $z_{78}$ as though the lines 7 and 8 were the adjacent lines of a self-energy subdiagram, and assigned the halved value to $z_{7}=z_{78} / 2$. Thus the integral was evaluated with 10 dimensions. This resulted in the wrong value of $X 024$ in the previous calculation.

The correction of this error changes the $X 024$ integral from $-6.0902(246)$ to $-7.3480(139)$. We carefully examined the mapping parts of the other 388 integrals and found no error. The new numerical values of these 388 integrals are consistent with the previous results as shown in Table I that lists the results of old evaluation, except that the wrong value of $X 024$ is removed, the results of new independent evaluation, and the statistical combination of these two sets of results.

\section{RESIDUAL RENORMALIZATION TERMS}

To obtain the physical contribution of Set V, the standard on-shell renormalization prescription has to be followed. The $\boldsymbol{K}$-operation prescription adopted in the numerical evaluation, however, is different from that, and thus must be adjusted for the difference. The IR subtraction terms introduced by $\boldsymbol{R}$ - and/or $\boldsymbol{I}$-operations to make each diagram IR-finite should also be restored. The IR divergences that reside in these two types of adjustments compensate with each other among several diagrams of Set V, leaving finite terms behind. This residual renormalization leads to the expression of the physical contribution $A_{1}^{(10)}[\mathrm{Set} \mathrm{V}]$ given by

$$
\begin{aligned}
A_{1}^{(10)}[\mathrm{Set} \mathrm{V}]= & \Delta M_{10}+\Delta M_{8}\left(-7 \Delta L B_{2}\right)+\Delta M_{6}\left\{-5 \Delta L B_{4}+20\left(\Delta L B_{2}\right)^{2}\right\} \\
& +\Delta M_{4}\left\{-3 \Delta L B_{6}+24 \Delta L B_{4} \Delta L B_{2}-28\left(\Delta L B_{2}\right)^{3}\right\}+\Delta M_{4}\left(2 \Delta d m_{4} \Delta L_{2^{*}}\right) \\
& +M_{2}\left\{-\Delta L B_{8}+8 \Delta L B_{6} \Delta L B_{2}-28 \Delta L B_{4}\left(\Delta L B_{2}\right)^{2}+4\left(\Delta L B_{4}\right)^{2}+14\left(\Delta L B_{2}\right)^{4}\right\} \\
& +M_{2} \Delta d m_{6}\left(2 \Delta L_{2^{*}}\right)+M_{2} \Delta d m_{4}\left(-16 \Delta L B_{2} \Delta L_{2^{*}}-2 \Delta d m_{2^{*}} \Delta L_{2^{*}}+\Delta L_{4^{*}}\right) .
\end{aligned}
$$

$\Delta M_{n}, \Delta L B_{n}, \Delta d m_{n}$ are obtained from the magnetic moment amplitudes, the sum of vertex and wave-function renormalization constants, and the mass renormalization constants, respectively, of the $n$ th-order diagrams without a fermion loop. Here the renormalization condition is the standard on-shell one. An asterisk $(*)$ indicates that the quantity be derived from diagrams having a two-point vertex insertion. UV divergences of all quantities are removed by the $\boldsymbol{K}$-operations, while IR divergences are removed by the $\boldsymbol{R}$ - and/or $\boldsymbol{I}$-operations. The overall IR divergences in the vertex and wave-function renormalization constants $L_{n}$ and $B_{n}$, respectively, cannot be handled by $\boldsymbol{R}$ - nor $\boldsymbol{I}$-operations. They are, however, canceled when two are summed because of the Ward-Takahashi identity. The precise definitions of these symbols are given in Refs. [52,55]. Their numerical values are listed in Table II, where most of the numbers are copied from Table II of Ref. [34]. Now that the eighth-order contribution has been confirmed [32], it implies that the residual renormalization constants used to derive (10) should also be correct. The value $\Delta M_{8}=1.73812(85)$ in the previous work is replaced by a new and more accurate value $\Delta M_{8}=$ 1.738467 (20) derived from the almost exact eighth-order result Eq. (5) of Ref. [32].

Among many quantities appearing in (27), only three, $\Delta L_{4 *}, \Delta M_{10}$, and $\Delta L B_{8}$, have not been independently checked. The quantity $\Delta L_{4^{*}}$ is derived from the fourthorder vertex diagrams with a two-point vertex insertion and was obtained by carrying out small and quick numerical calculations. The finite magnetic moment amplitude of tenth order $\Delta M_{10}$ is obtained by summing up the last columns of Table I. The finite part of the vertex and wavefunction renormalization constants of eighth order $\Delta L B_{8}$ is also obtained by numerical means.

TABLE II. Residual renormalization constants used to calculate $A_{1}^{(10)}\left[\right.$ Set V]. The $\Delta M_{n}, \Delta L B_{n}$, and $\Delta d m_{n}$ are the sum of the finite magnetic moment amplitudes, the sum of the finite parts of vertex and wave-function renormalization constants, and the sum of the finite parts of the mass-renormalization constants, respectively, all derived from the $n$th order-diagrams without a fermion loop of the QED perturbation theory. $\Delta M_{10}$ is newly calculated in this work. $\Delta M_{8}$ is derived from the near-exact result Eq. (5) of Ref. [32]. Other finite integrals are copied from the previous work Table II of Ref. [34].

\begin{tabular}{ll}
\hline \hline Integral & Value (Error) \\
\hline$\Delta M_{10}$ & $2.350(192)$ \\
$\Delta M_{8}$ & $1.738467(20)$ \\
$\Delta M_{6}$ & $0.4258135(30)$ \\
$\Delta M_{4}$ & $0.030833612 \ldots$ \\
$M_{2}$ & 0.5 \\
$\Delta L B_{8}$ & $2.0504(86)$ \\
$\Delta L B_{6}$ & $0.100801(43)$ \\
$\Delta L B_{4}$ & $0.0279171(61)$ \\
$\Delta L B_{2}$ & 0.75 \\
$\Delta L_{4^{*}}$ & $-0.459051(62)$ \\
$\Delta L_{2^{*}}$ & -0.75 \\
$\Delta d m_{6}$ & $-2.340815(55)$ \\
$\Delta d m_{4}$ & $1.9063609(90)$ \\
$\Delta d m_{2^{*}}$ & -0.75 \\
\hline \hline
\end{tabular}


TABLE III. Integrals contributing to the residual renormalization constants $\Delta L B_{8}$. The 4710 -dimensional integrals derived from the eighth-order vertex and self-energy renoromalization constants are evaluated by VEGAS. The second column shows the diagarm representation by photon labels attached to the fermion line of a self-energy diagram. For $L B 04, L B 11$, $L B 12, L B 16, L B 17, L B 18, L B 29$, and $L B 30$, double-double precision for real numbers is partially used. Others are evaluated in double precision.

\begin{tabular}{|c|c|c|}
\hline Integral & Expression & Value (Error) \\
\hline$L B 01$ & $a b a c b d c d$ & $-0.19096(80)$ \\
\hline$L B 02$ & $a b a c b d d c$ & 0.60407 (177) \\
\hline$L B 03$ & $a b a c c d b d$ & $0.42796(150)$ \\
\hline$L B 04$ & $a b a c c d d b$ & $-1.00569(198)$ \\
\hline$L B 05$ & $a b a c d b c d$ & $-0.81962(71)$ \\
\hline$L B 06$ & $a b a c d b d c$ & $-0.10117(96)$ \\
\hline$L B 07$ & $a b a c d c b d$ & $-0.16526(94)$ \\
\hline$L B 08$ & $a b a c d c d b$ & $0.47320(185)$ \\
\hline$L B 09$ & $a b a c d d b c$ & $0.77321(187)$ \\
\hline$L B 10$ & $a b a c d d c b$ & $-0.48403(177)$ \\
\hline$L B 11$ & $a b b c a d d c$ & $-0.40321(185)$ \\
\hline$L B 12$ & $a b b c c d d a$ & 0.60668 (189) \\
\hline$L B 13$ & $a b b c d a c d$ & 0.90458 (109) \\
\hline$L B 14$ & $a b b c d a d c$ & $0.16456(142)$ \\
\hline$L B 15$ & $a b b c d c a d$ & 0.13619 (136) \\
\hline$L B 16$ & $a b b c d c d a$ & -1.01314 (239) \\
\hline$L B 17$ & $a b b c d d a c$ & $-0.97511(193)$ \\
\hline$L B 18$ & $a b b c d d c a$ & $3.20432(195)$ \\
\hline$L B 19$ & $a b c a d b c d$ & $0.21777(18)$ \\
\hline$L B 20$ & $a b c a d b d c$ & $-0.44527(40)$ \\
\hline$L B 21$ & $a b c a d c b d$ & -0.16893 \\
\hline$L B 22$ & $a b c a d c d b$ & $0.17741(40)$ \\
\hline$L B 23$ & $a b c a d d b c$ & $0.60213(115)$ \\
\hline$L B 24$ & $a b c a d d c b$ & $0.06722(87)$ \\
\hline$L B 25$ & $a b c b d a d c$ & $0.01343(23)$ \\
\hline$L B 26$ & $a b c b d c d a$ & $0.35136(94)$ \\
\hline$L B 27$ & $a b c b d d a c$ & $0.26002(130)$ \\
\hline$L B 28$ & $a b c b d d c a$ & $-0.85851(231)$ \\
\hline$L B 29$ & $a b c c d d a b$ & $-0.49148(145)$ \\
\hline$L B 30$ & $a b c c d d b a$ & $0.49681(217)$ \\
\hline$L B 31$ & $a b c d a b c d$ & $-0.83179(28)$ \\
\hline$L B 32$ & $a b c d a b d c$ & 0.38767 (29) \\
\hline$L B 33$ & $a b c d a c b d$ & $0.25953(11)$ \\
\hline$L B 34$ & $a b c d a c d b$ & $-0.31291(30)$ \\
\hline$L B 35$ & $a b c d a d b c$ & $-0.36911(36)$ \\
\hline$L B 36$ & $a b c d a d c b$ & $0.07706(48)$ \\
\hline$L B 37$ & $a b c d b a d c$ & $-0.17446(21)$ \\
\hline$L B 38$ & $a b c d b c d a$ & $0.08696(37)$ \\
\hline$L B 39$ & $a b c d b d a c$ & $0.22033(33)$ \\
\hline$L B 40$ & $a b c d b d c a$ & $0.17934(183)$ \\
\hline$L B 41$ & $a b c d c d a b$ & $0.29211(58)$ \\
\hline$L B 42$ & $a b c d c d b a$ & $-0.31087(91)$ \\
\hline$L B 43$ & $a b c d d a b c$ & $0.62911(39)$ \\
\hline$L B 44$ & $a b c d d a c b$ & $-0.15460(108)$ \\
\hline$L B 45$ & $a b c d d b c a$ & $-0.29617(106)$ \\
\hline$L B 46$ & $a b c d d c a b$ & $-0.32517(66)$ \\
\hline$L B 47$ & $a b c d d c b a$ & 0.33483 (116) \\
\hline
\end{tabular}

To obtain the value of $\Delta L B_{8}$, we have to deal with 518 eighth-order vertex diagrams and 74 self-energy diagrams. The Ward-Takahashi identity relates seven vertex diagrams to one self-energy diagram. Because of the time-reversal symmetry, the number of self-energy-like diagrams is reduced to 47. Extraction of the finite part of the renormalization constants in a form of Feynman-parametric integral from a self-energy-like diagram was carried out by using an automatic code generator similar to GENCODE $N$. The validity of the code generator has been checked for the sixth-order quantity $\Delta L B_{6}$, which leads to the correct eighth-order contribution (10). The divergence structure of the 47 finite integrals of $\Delta L B_{8}$ and the 8 integrals of $\Delta L B_{6}$ can be symbolically expressed as shown by Eq. (B14) of Ref. [34]. The 47 integrals, which are 10dimensional integrals, are then numerically evaluated by VEGAS. Their values are listed in Table III.

Collecting all these results and substituting numerical values into Eq. (27), we obtain (14) as the best estimate for the Set V contribution to $a_{e}$.

\section{CONCLUSION}

We have reevaluated all 389 integrals representing the tenth-order Set V diagrams. The error in the integral X024 was identified and corrected. For the other 388 integrals, different sets of integration variables were prepared and independent numerical evaluation was performed. More statistics have been accumulated which enabled us to obtain the more reliable and accurate numerical results. Together with the semianalytic eighth-order result, the uncertainty of the QED contribution to the electron anomalous magnetic moment $\left(a_{e}\right)$ has been reduced to the same order of uncertainty as that of the hadronic contribution.

\section{ACKNOWLEDGMENTS}

We thank Dr. F. Jegerlehner for providing us the details of his calculation on the hadronic contribution to $a_{e}$ prior to its publication. We also thank Dr. B. Taylor for notifying us of the new CODATA adjustment of fundamental physical constants. We thank Dr. A. Kataev, Dr. M. Hoferichter, and Dr. D. Nomura for communication. T. K.'s work is supported in part by the U.S. National Science Foundation under Grant No. NSF-PHY-1316222. M. N.'s work is supported in part by the JSPS Grant-in-Aid for Scientific Research (C) 16K05338. Numerical calculations were conducted on the supercomputing systems on RICC, HOKUSAI-GreatWave, and HOKUSAI-BigWaterfall at RIKEN.

\section{APPENDIX: UPDATES FOR THE MUON $g-2$}

The world average of the measurements of the muon anomalous magnetic moment $\left(a_{\mu}\right)$ is dominated by the BNL experiments and is given as [44,57] 


$$
a_{\mu}(\text { expt. })=116592089(63) \times 10^{-11}[0.5 \mathrm{ppm}] .
$$

A new experiment is currently being prepared at Fermilab using the storage ring that was used for the previous BNL measurements and was moved from BNL [58]. The first result of the Fermilab E989 experiment is expected to be announced in Spring 2018 [59]. Another new experiment is being prepared at J-PARC [60]. Cold muon beam and a storage ring with $66 \mathrm{~cm}$ diameter are used for this E34 experiment [61]. Two measurements with completely different apparatus will provide us far deeper insight on the physics of muon $g-2$.

The QED contribution to $a_{\mu}$ is affected by the improved values of the mass-independent eighth- and tenth-order terms and by the new values of the fine-structure constant. Two independent determinations of $\alpha$ lead to two values of the QED contribution of $a_{\mu}$. By using the $\alpha$ from the Rbatom experiment (19) or that from the electron $g-2(21)$, the QED contribution of $a_{\mu}$ is given as

$$
\begin{aligned}
a_{\mu}(\mathrm{QED}, \alpha(\mathrm{Rb}))= & 1165847189.71(7)(17)(6)(72) \\
& \times 10^{-12},
\end{aligned}
$$

or

$$
\begin{aligned}
a_{\mu}\left(\mathrm{QED}, \alpha\left(a_{e}\right)\right)= & 1165847188.41(7)(17)(6)(28) \\
& \times 10^{-12},
\end{aligned}
$$

respectively, where the assigned uncertainties are due to the lepton-mass ratios, the numerical evaluation of the eighthorder QED term, the numerical evaluation of the tenthorder QED term, and the fine-structure constant from left to right. By now the entire eighth-order QED term has been cross-checked by two or more independent calculations. The mass-dependent eighth-order QED terms involving the electron or tau-lepton have been calculated by numerical means $[62,63]$ and the results are confirmed by analytic calculation $[15,64,65]$. The QED predictions (A2) and (A3) are changed by $+0.20 \times 10^{-12}$ and $-0.05 \times 10^{-12}$, respectively, from those given in Ref. [63], which does not affect comparison of theory and measurement of $a_{\mu}$.

To compare theory of $a_{\mu}$ to measurement, the hadronic and electroweak contributions must be added to the dominant QED contribution (A2) or (A3). The hadronic vacuum-polarization (HVP) contribution has been calculated by three groups based on newest measurements of the hadronic cross section. The LO-HVP contribution is found in Refs. [39,66,67]. Recently a remarkable progress has been achieved in lattice calculation of the LO-HVP contribution [68,69], although it is not yet competitive in precision to the dispersion calculation based on measured hadronic cross sections. Reevaluation of the NLO-HVP contribution by means of the dispersion integral is given in Refs. $[39,66]$. The NNLO-HVP contribution is given in Ref. [70]. All the HVP contributions are summarized as

$$
\begin{gathered}
a_{\mu}(\mathrm{HVP}, \mathrm{LO})= \begin{cases}689.46(3.25) \times 10^{-10} & {[39]} \\
692.23(2.54) \times 10^{-10} & {[66],} \\
693.1(3.4) \times 10^{-10} & {[67]}\end{cases} \\
a_{\mu}(\mathrm{HVP}, \mathrm{NLO})=\left\{\begin{array}{ll}
-9.927(0.067) \times 10^{-10} & {[39]} \\
-9.83(0.04) \times 10^{-10} & {[66]}
\end{array},\right. \\
a_{\mu}(\mathrm{HVP}, \mathrm{NNLO})=1.24(0.01) \times 10^{-10}
\end{gathered}
$$

The largest uncertainty of the theory of $a_{\mu}$ comes from the hadronic light-by-light (HLbL) contribution. Various hadron models have been used to compute it. The widely accepted value of the LO-HLbL term is $10.5(2.6) \times 10^{-10}$ [71] or $11.6(4.0) \times 10^{-10}$ [72]. Both cover almost all model-dependent results [73-80]. Recently, the axial meson contribution to the HLbL has been revised [81], which makes the LO-HLbL contribution smaller. Other revisions have been made for the tensor-exchange contribution [81] and for the $\pi^{0}$ exchange contribution based on the lattice calculation [82]. By collecting these modifications, the LO-HLbL becomes [83]

$$
a_{\mu}(\mathrm{HLbL}, \mathrm{LO})=10.0(2.9) \times 10^{-10} .
$$

The NLO-HLbL term was estimated to be [84]

$$
a_{\mu}(\mathrm{HLbL}, \mathrm{NLO})=0.3(0.2) \times 10^{-10} .
$$

In the last few years, lattice QCD calculation of the HLbL contribution has become feasible [85-89]. Though the systematic uncertainty has not yet been determined, Ref. [87] gives the lattice QCD result as

$$
a_{\mu}(\mathrm{HLbL}, \mathrm{LO})=5.35(1.35) \times 10^{-10},
$$

where the uncertainty is due to statistics only.

The data-driven dispersion approach to HLbL similar to but far more complicated than that to HVP has been formulated $[90,91]$. It is a very promising method though it requires long and difficult theoretical works [92,93]. The contribution from the two- $\pi$ intermediate states has been recently determined [94], which is more accurate than that determined using the hadronic models.

For the electroweak contribution, up to two-loop calculations are known $[95,96]$. The two-loop weak correction was reevaluated using the latest measured mass of the Higgs boson [97] and the total contribution becomes

$$
a_{\mu}(\text { Weak })=15.36(10) \times 10^{-10} .
$$

The theoretical prediction of $a_{\mu}$ are obtained by summing up the QED, hadronic, and weak contributions. We use the model calculation (A7) for the LO-HLbL contribution. The three values of the LO-HVP estimates listed in Eq. (A4) lead the theoretical prediction of $a_{\mu}$ as 
$a_{\mu}($ theory $)= \begin{cases}116591783(51) \times 10^{-11} & {[39]} \\ 116591812(47) \times 10^{-11} & {[66]} \\ 116591820(52) \times 10^{-11} & {[67]}\end{cases}$

(A11)
The difference between measurement and theory ranges from $269 \sim 306 \times 10^{-11}$ corresponding to $3.3 \sim 3.8 \sigma$ discrepancy.
[1] P. Kusch and H. M. Foley, Phys. Rev. 72, 1256 (1947).

[2] J. S. Schwinger, Phys. Rev. 73, 416 (1948).

[3] D. Hanneke, S. Fogwell, and G. Gabrielse, Phys. Rev. Lett. 100, 120801 (2008).

[4] D. Hanneke, S. Fogwell Hoogerheide, and G. Gabrielse, Phys. Rev. A 83, 052122 (2011).

[5] R. S. Van Dyck, P. B. Schwinberg, and H. G. Dehmelt, Phys. Rev. Lett. 59, 26 (1987).

[6] S. Fogwell Hoogerheide, J. C. Dorr, E. Novitski, and G. Gabrielse, Rev. Sci. Instrum. 86, 053301 (2015).

[7] H. H. Elend, Phys. Lett. 20, 682 (1966).

[8] M. A. Samuel and G.-w. Li, Phys. Rev. D 44, 3935 (1991).

[9] G. Li, R. Mendel, and M. A. Samuel, Phys. Rev. D 47, 1723 (1993).

[10] S. Laporta and E. Remiddi, Phys. Lett. B 301, 440 (1993).

[11] S. Laporta, Nuovo Cimento Soc. Ital. Fis. 106A, 675 (1993).

[12] M. Passera, Phys. Rev. D 75, 013002 (2007).

[13] T. Kinoshita and M. Nio, Phys. Rev. D 73, 053007 (2006).

[14] A. L. Kataev, Phys. Rev. D 86, 013010 (2012).

[15] A. Kurz, T. Liu, P. Marquard, and M. Steinhauser, Nucl. Phys. B879, 1 (2014).

[16] T. Aoyama, M. Hayakawa, T. Kinoshita, M. Nio, and N. Watanabe, Phys. Rev. D 78, 053005 (2008).

[17] T. Aoyama, M. Hayakawa, T. Kinoshita, and M. Nio, Phys. Rev. D 78, 113006 (2008).

[18] T. Aoyama, K. Asano, M. Hayakawa, T. Kinoshita, M. Nio, and N. Watanabe, Phys. Rev. D 81, 053009 (2010).

[19] T. Aoyama, M. Hayakawa, T. Kinoshita, and M. Nio, Phys. Rev. D 82, 113004 (2010).

[20] T. Aoyama, M. Hayakawa, T. Kinoshita, and M. Nio, Phys. Rev. D 83, 053003 (2011).

[21] T. Aoyama, M. Hayakawa, T. Kinoshita, and M. Nio, Phys. Rev. D 83, 053002 (2011).

[22] T. Aoyama, M. Hayakawa, T. Kinoshita, and M. Nio, Phys. Rev. D 84, 053003 (2011).

[23] T. Aoyama, M. Hayakawa, T. Kinoshita, and M. Nio, Phys. Rev. D 85, 033007 (2012).

[24] T. Aoyama, M. Hayakawa, T. Kinoshita, and M. Nio, Phys. Rev. D 85, 093013 (2012).

[25] T. Aoyama, M. Hayakawa, T. Kinoshita, and M. Nio, Phys. Rev. Lett. 109, 111807 (2012).

[26] P. A. Baikov, A. Maier, and P. Marquard, Nucl. Phys. B877, 647 (2013).

[27] A. Petermann, Helv. Phys. Acta 30, 407 (1957).

[28] C. M. Sommerfield, Ann. Phys. (N.Y.) 5, 26 (1958).

[29] S. Laporta and E. Remiddi, Phys. Lett. B 379, 283 (1996).

[30] T. Kinoshita, Phys. Rev. Lett. 75, 4728 (1995).

[31] K. Melnikov and T. van Ritbergen, Phys. Rev. Lett. 84, 1673 (2000).
[32] S. Laporta, Phys. Lett. B 772, 232 (2017).

[33] T. Kinoshita and W. B. Lindquist, Phys. Rev. Lett. 47, 1573 (1981).

[34] T. Aoyama, M. Hayakawa, T. Kinoshita, and M. Nio, Phys. Rev. D 91, 033006 (2015); 96, 019901(E) (2017).

[35] P. Marquard, A. V. Smirnov, V. A. Smirnov, M. Steinhauser, and D. Wellmann, in International Workshop on $e+e-$ Collisions from Phi to Psi (PHIPSI17) Mainz, Germany, June 26-29, 2017 (2017), arXiv:1708.07138.

[36] S. Volkov, Phys. Rev. D 96, 096018 (2017).

[37] M. Caffo, S. Turrini, and E. Remiddi, Nucl. Phys. B141, 302 (1978).

[38] G. P. Lepage, J. Comput. Phys. 27, 192 (1978).

[39] F. Jegerlehner, arXiv:1711.06089.

[40] M. Ablikim et al. (BESIII Collaboration), Phys. Lett. B 753, 629 (2016).

[41] V. V. Anashin et al., Phys. Lett. B 770, 174 (2017).

[42] D. Nomura and T. Teubner, Nucl. Phys. B867, 236 (2013).

[43] B. Taylor and F. Jegerlehner (private communication).

[44] P. J. Mohr, D. B. Newell, and B. N. Taylor, Rev. Mod. Phys. 88, 035009 (2016).

[45] P. J. Mohr, D. B. Newell, B. N. Taylor, and E. Tiesinga, Metrologia (to be published), https://doi.org/10.1088/16817575/aa99bc.

[46] R. Bouchendira, P. Clade, S. Guellati-Khelifa, F. Nez, and F. Biraben, Phys. Rev. Lett. 106, 080801 (2011).

[47] G. Audi, M. Wang, A. Wapstra, F. Kondev, M. MacCormick, X. Xu, and B. Pfeiffer, Chin. Phys. C 36, 1287 (2012).

[48] M. Wang, G. Audi, A. Wapstra, F. Kondev, M. MacCormick, X. Xu, and B. Pfeiffer, Chin. Phys. C 36, 1603 (2012).

[49] P. J. Mohr, B. N. Taylor, and D. B. Newell, Rev. Mod. Phys. 84, 1527 (2012).

[50] P. Cvitanović and T. Kinoshita, Phys. Rev. D 10, 3978 (1974).

[51] T. Kinoshita, in Quantum Electrodynamics, edited by T. Kinoshita (World Scientific, Singapore, 1990), p. 218.

[52] T. Aoyama, M. Hayakawa, T. Kinoshita, and M. Nio, Nucl. Phys. B740, 138 (2006).

[53] W. Zimmermann, Commun. Math. Phys. 15, 208 (1969).

[54] P. Cvitanović and T. Kinoshita, Phys. Rev. D 10, 3991 (1974).

[55] T. Aoyama, M. Hayakawa, T. Kinoshita, and M. Nio, Nucl. Phys. B796, 184 (2008).

[56] Y. Hida, X. S. Li, and D. H. Bailey, in Proceedings. 15th IEEE Symposium on Computer Arithmetic ARITH-15 2001 (IEEE Computer Society, Los Alamitos, CA, USA, 2001), p. 155 .

[57] G. W. Bennett et al. (Muon g-2 Collaboration), Phys. Rev. D 73, 072003 (2006). 
[58] J. Grange et al. (Muon g-2 Collaboration), arXiv: 1501.06858.

[59] A. Chapelain (Muon g-2 Collaboration), EPJ Web Conf. 137, 08001 (2017).

[60] H. Iinuma (J-PARC New g-2/EDM experiment Collaboration), J. Phys. Conf. Ser. 295, 012032 (2011).

[61] M. Otani (E34 Collaboration), JPS Conf. Proc. 8, 025008 (2015).

[62] T. Kinoshita and M. Nio, Phys. Rev. D 70, 113001 (2004).

[63] T. Aoyama, M. Hayakawa, T. Kinoshita, and M. Nio, Phys. Rev. Lett. 109, 111808 (2012).

[64] A. Kurz, T. Liu, P. Marquard, A. V. Smirnov, V. A. Smirnov, and M. Steinhauser, Phys. Rev. D 93, 053017 (2016).

[65] A. Kurz, T. Liu, P. Marquard, A. V. Smirnov, V. A. Smirnov, and M. Steinhauser, Phys. Rev. D 92, 073019 (2015).

[66] T. Teubner (2017), international workshop on $e+e-$ collisions from Phi to Psi 2017, 26-29 June, 2017, Mainz, Germany, URL https://indico.mitp.uni-mainz.de/event/86/ contribution/17/material/slides/0.pdf.

[67] M. Davier, A. Hoecker, B. Malaescu, and Z. Zhang, Eur. Phys. J. C 77, 827 (2017).

[68] B. Chakraborty, C. T. H. Davies, P. G. de Oliveira, J. Koponen, G. P. Lepage, and R. S. Van de Water, Phys. Rev. D 96, 034516 (2017).

[69] T. Blum, P. A. Boyle, T. Izubuchi, L. Jin, A. Jüttner, C. Lehner, K. Maltman, M. Marinkovic, A. Portelli, and M. Spraggs, Phys. Rev. Lett. 116, 232002 (2016).

[70] A. Kurz, T. Liu, P. Marquard, and M. Steinhauser, Phys. Lett. B 734, 144 (2014).

[71] J. Prades, E. de Rafael, and A. Vainshtein, in Lepton Dipole Moments, edited by B.L. Roberts and W. J. Marciano (World Scientific, Singapore, 2009), p. 303.

[72] F. Jegerlehner and A. Nyffeler, Phys. Rep. 477, 1 (2009).

[73] J. Bijnens, E. Pallante, and J. Prades, Phys. Rev. Lett. 75, 1447 (1995); 75, 3781(E) (1995).

[74] J. Bijnens, E. Pallante, and J. Prades, Nucl. Phys. B474, 379 (1996).

[75] M. Hayakawa, T. Kinoshita, and A. I. Sanda, Phys. Rev. Lett. 75, 790 (1995).

[76] M. Hayakawa, T. Kinoshita, and A. I. Sanda, Phys. Rev. D 54, 3137 (1996).
[77] M. Hayakawa and T. Kinoshita, Phys. Rev. D 57, 465 (1998); 66, 019902(E) (2002).

[78] K. Melnikov and A. Vainshtein, Phys. Rev. D 70, 113006 (2004).

[79] J. Bijnens and J. Prades, Mod. Phys. Lett. A 22, 767 (2007).

[80] A. Nyffeler, Phys. Rev. D 79, 073012 (2009).

[81] V. Pauk and M. Vanderhaeghen, Eur. Phys. J. C 74, 3008 (2014).

[82] A. Gérardin, H. B. Meyer, and A. Nyffeler, Phys. Rev. D 94, 074507 (2016).

[83] F. Jegerlehner, arXiv:1705.00263.

[84] G. Colangelo, M. Hoferichter, A. Nyffeler, M. Passera, and P. Stoffer, Phys. Lett. B 735, 90 (2014).

[85] J. Green, O. Gryniuk, G. von Hippel, H. B. Meyer, and V. Pascalutsa, Phys. Rev. Lett. 115, 222003 (2015).

[86] N. Asmussen, J. Green, H. B. Meyer, and A. Nyffeler, Proc. Sci., LATTICE2016 (2016) 164.

[87] T. Blum, N. Christ, M. Hayakawa, T. Izubuchi, L. Jin, C. Jung, and C. Lehner, Phys. Rev. Lett. 118, 022005 (2017).

[88] T. Blum, N. Christ, M. Hayakawa, T. Izubuchi, L. Jin, C. Jung, and C. Lehner, Phys. Rev. D 96, 034515 (2017).

[89] A. Gérardin, J. Green, O. Gryniuk, G. von Hippel, H. B. Meyer, V. Pascalutsa, and H. Wittig, arXiv:1712.00421 [Phys. Rev. D (to be published)].

[90] G. Colangelo, M. Hoferichter, M. Procura, and P. Stoffer, J. High Energy Phys. 09 (2014) 091.

[91] V. Pauk and M. Vanderhaeghen, Phys. Rev. D 90, 113012 (2014).

[92] G. Colangelo, M. Hoferichter, M. Procura, and P. Stoffer, J. High Energy Phys. 09 (2015) 074.

[93] G. Colangelo, M. Hoferichter, B. Kubis, M. Procura, and P. Stoffer, Phys. Lett. B 738, 6 (2014).

[94] G. Colangelo, M. Hoferichter, M. Procura, and P. Stoffer, J. High Energy Phys. 04 (2017) 161.

[95] K. Fujikawa, B. Lee, and A. Sanda, Phys. Rev. D 6, 2923 (1972).

[96] A. Czarnecki, W. J. Marciano, and A. Vainshtein, Phys. Rev. D 67, 073006 (2003); 73, 119901(E) (2006).

[97] C. Gnendiger, D. Stöckinger, and H. Stöckinger-Kim, Phys. Rev. D 88, 053005 (2013). 